\title{
Precancer: Mutant clones in normal epithelium outcompete and eliminate esophageal micro-tumors
}

\author{
B. Colom ${ }^{1}$, A. Herms ${ }^{1}$, M.W.J. Hall ${ }^{1,2}$, S.C. Dentro ${ }^{1,3}$, C. King ${ }^{1}$, R.K. Sood ${ }^{1}$, M.P. Alcolea, ${ }^{4,5}$, G. \\ Piedrafita $^{1,6}$, D. Fernandez-Antoran ${ }^{1}$, S.H. Ong ${ }^{1}$, J.C. Fowler ${ }^{1}$, K.T. Mahbubani ${ }^{7}$, K. Saeb-

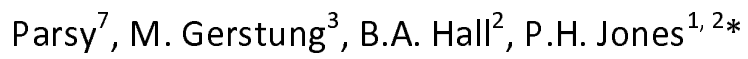

Affiliations:

${ }^{1}$ Wellcome Sanger Institute, Hinxton CB10 1SA, UK.

2 Department of Medical Physics and Biomedical Engineering, University College London, Gower Street, London WC1E 6BT, UK.

${ }^{3}$ European Molecular Biology Laboratory, European Bioinformatics Institute, Cambridge CB10 1SD, UK.

4 Wellcome-MRC Cambridge Stem Cell Institute, Jeffrey Cheah Biomedical Centre, Cambridge Biomedical Campus, University of Cambridge, Cambridge, CB2 OAW.

${ }^{5}$ Department of Oncology, University of Cambridge, Hutchison/MRC Research Centre, Hills Road, Cambridge Biomedical Campus, Cambridge CB2 OXZ, UK.

${ }^{6}$ Spanish National Cancer Research Centre (CNIO), Madrid 28029, Spain.

${ }^{7}$ Department of Surgery and Cambridge NIHR Biomedical Research Centre, Biomedical Campus, Cambridge CB2 OQQ

* Correspondence to: pj3@sanger.ac.uk 


\section{Summary}

Human epithelial tissues accumulate cancer-driver mutations with age $\mathrm{e}^{1-7}$, yet tumor formation remains rare. The positive selection of these mutations argues they alter the behavior and fitness of proliferating cells ${ }^{8-10}$. Hence, normal adult tissues become a patchwork of mutant clones competing for space and survival, with the fittest clones expanding by eliminating their less-competitive neighbors ${ }^{9-12}$. However, little is known about how such dynamic competition in normal epithelia impacts early tumorigenesis. Here we show that the majority of newly formed esophageal tumors are eliminated through competition with mutant clones in the surrounding normal epithelium. We followed the fate of microscopic tumors in a mouse model of esophageal carcinogenesis. Most neoplasms are rapidly lost despite no indication of tumor cell death, decreased proliferation, or an anti-tumor immune response. Deep-sequencing of 10-day and 1-yearold tumors shows evidence of genetic selection on the surviving neoplasms. Induction of highly competitive clones in transgenic mice increased tumor removal, while pharmacologically inhibiting clonal competition reduced tumor loss. The results are consistent with a model where survival of early neoplasms depends on their competitive fitness relative to that of mutant clones in the adjacent normal tissue. We have identified an unexpected anti-tumorigenic role for mutant clones in normal epithelium by purging early neoplasms through cell competition, thereby preserving tissue integrity. 


\section{Main text}

Somatic mutations in cancer-driver genes are frequent in phenotypically normal adult human epithelia such as that of the esophagus, skin, endometrium, lung and colon ${ }^{1-7}$. Some of these mutations are more prevalent in normal epithelium than neoplastic lesions, leading to the suggestion that mutant clones in the non-neoplastic epithelium might have an unanticipated protective role against cancer $^{2,5}$. Defining how mutant clones in normal epithelium and early neoplastic lesions compete for space and survival may be critical to understand the initial stages of tumorigenesis. However, despite the potential implications for cancer development, our knowledge of how mutant clones in normal tissues and tumors interact is limited.

Here we investigated this issue in the mouse esophageal epithelium (EE). This tissue consists of a single basal layer of progenitor cells that periodically divide and eventually stratify upwards forming a multi-layer of differentiated cells that are finally shed (Extended Data Fig. 1a). Dividing cells have an equal probability of generating progenitor and differentiating daughters, a balance that maintains tissue homeostasis (Extended Data Fig. 1b and Supplementary information) ${ }^{13,14}$. Some mutations transiently tilt this balance towards higher production of progenitor cells, thus conferring a competitive advantage over their less fit neighbors (Extended Data Fig. 1c) ${ }^{9,12}$.

Administration of diethylnitrosamine (DEN), a mutagen present in tobacco smoke, in drinking water induces mutations in the epithelial cells of the mouse esophagus ${ }^{11}$. Cells with competitive "winner" mutations form clones that expand laterally in the proliferative basal cell layer until they collide with other mutants of similar fitness, at which point they return towards neutral drift (Extended Data Fig. 1d and Supplementary information) ${ }^{11}$. With time, mutant clones in the phenotypically normal mouse EE undergo genetic selection as they compete for the limited space (Supplementary information), and generate a mutational landscape similar to that found in the aging human normal esophagus ${ }^{11}$. In addition, DEN treatment leads to the formation of discrete tumors scattered within this mutant patchwork ${ }^{9,15}$, which makes this an ideal model to study interactions between mutant clones in normal and neoplastic epithelium. 


\section{Most EE tumors are lost over time}

1 year after DEN treatment mice exhibit an average of one tumor per $E^{15}$. To study earlier steps in tumor development here we collected esophagi at different time-points, from as early as 10 days up to 18 months post-DEN treatment (Fig. 1a). After tissue collection the entire EE was 3D-imaged at single cell resolution by confocal microscopy, following immuno-staining of tumors with the stress marker protein KRT6 ${ }^{15}$ (Fig. 1b and Extended Data Fig. 2a-c). This technique allowed us to detect hundreds of 'micro-neoplasms' per esophagus at 10 days post-DEN withdrawal. These were as small as $\sim 50 \mu \mathrm{m}$ in diameter and comprising as little as $\sim 20$ cells, with histological features of focal hyperplasia, (Fig. 1b-d). Remarkably, the number of tumors in the EE fell rapidly during the following months and stabilized after this initial phase (Fig. 1d and Supplementary Table 1). Persisting tumors kept growing and developed features of dysplasia, with angiogenesis in the underlying submucosa and leukocyte infiltration (Fig. 1b, c, e; Extended Data Fig. 2c-e and Supplementary Table 1). We conclude that most of the early EE tumors generated by DEN treatment are resolved soon after they are formed (Fig. 1f).

\section{Sequencing of newly formed and surviving tumors}

To gain insight into how this process may occur we next characterized and compared the mutational landscapes of newly formed and surviving tumors, collected at 10 days and 1 year post-DEN treatment, respectively (Fig. 2a; Extended Data Figs. 3 and 4a). Deeptargeted sequencing, with a median on-target coverage 236x, of 192 genes implicated in epithelial carcinogenesis identified a total of 11,149 mutations from 141 tumors (Fig. 2b, Extended Data Fig. 4b and Supplementary Tables 2 and 3). The mutational spectrum comprised mainly $T>A / A>T, T>C / A>G$ and $C>T / G>A$ alterations, consistent with $D E N$ mutagenesis in mouse EE (Extended Data Fig. 4c) ${ }^{11}$. Functionally, most mutations were protein altering, with missense SNVs being the most common, a pattern that was maintained over time (Extended Data Fig. 4d-e). To test whether genetic selection was a feature of tumor survival we calculated the $\mathrm{dN} / \mathrm{dS}$ (non-synonymous-to synonymous) mutation ratios across all sequenced genes, with $\mathrm{dN} / \mathrm{dS}$ values over 1 indicating positive 
selection ${ }^{2,3,16}$. Mutant Notch1 and Trp53 were positively selected in 10 day neoplasms, whereas Atp2a2, Notch1, Notch2, Chuk and Adam10 were selected in the surviving tumors at 1 year (Fig. 2c and Supplementary Tables 4 and 5). No additional driver genes were identified by $\mathrm{dN} / \mathrm{dS}$ analysis of whole exome sequenced tumors (Extended Data Fig. 5; Supplementary Tables 6-7 and Supplementary information). Mutations in Atp2a2 and Notch1 dominated clonal selection within tumors, exhibiting the highest gain in $\mathrm{dN} / \mathrm{dS}$ values, number of mutations and estimated proportion of tumor area occupied by the mutant gene (Fig. 2 c-d and Extended Data Fig. 4f). Strikingly, over $98 \%$ of the persisting tumors at 1 year carried non-synonymous mutations in one or both of these genes (Extended Data Fig. 4g). Of note, whole exome and whole genome sequencing analysis showed that chromosomal alterations were negligible in these tumors, indicating that genome instability does not underpin tumor survival in our model (Extended Data Fig. 6 and Supplementary information). Together these results argue that the early generated EE neoplasms undergo genetic selection, a process that may influence their survival or elimination from the tissue.

\section{Mechanisms of early tumor loss}

We next sought to identify the mechanism(s) responsible for early tumor loss. Immunostaining of activated caspase 3, EdU (5-ethynyl-2? -deoxyuridine) short-term DNA incorporation assays and label-retaining experiments using Rosa26 $6^{\text {M2rtTA }} /$ TetO-HGFP mice, demonstrated that early tumors were not lost by apoptosis or abnormal proliferation of tumor cells (Fig. 3a-d, Extended Data Fig. 7a-c, Supplementary Table 8 and Supplementary information). We next explored whether the immune system could be eliminating these early neoplasms. However, although immune $\left(\mathrm{CD} 45^{+}\right)$cells were occasionally observed in close contact with early tumors, the density of immune cells within them was no higher than in the surrounding tissue (Extended Data Fig. 7d-j and Supplementary information). Furthermore, immune-deficient NOD.Cg-Prkdc $c^{s c i d} / 12 r^{t m 1 W_{j l}} / S z J$ mice, which lack the typical anti-tumor immune response, showed equivalent tumor formation and loss rates as compared to wild-type controls. These results argue that the immune system is neither implicated in the initiation or elimination of early tumors, and that tumor removal takes 
place prior these being detected by the immune system (Fig. 3e-f and Supplementary information).

\section{Mutant clones in normal EE remove tumors}

We next investigated whether competition with mutant clones in the surrounding normal EE may act as a selective pressure for early tumor survival. We have previously described how highly competitive mutant clones in the normal EE of DEN-treated mice eliminate their less-fit neighbors (Supplementary information) ${ }^{11}$. Thus, we hypothesized that a similar "survival of the fittest" competition mechanism could be responsible for the loss of early tumors (Fig. 4a and Supplementary information). To test this hypothesis, we analyzed whether induction of highly fit mutant clones in the normal epithelium altered early tumor survival (Extended Data Fig. 8a-b and Supplementary information). For this we used Ahcre ${ }^{E R T}$ Rosa26 ${ }^{\text {wt/DNM-GFP }}$ (MAML-Cre) mice, which carry a highly competitive inducible dominant negative mutant allele of Maml-1 (DN-Maml1) that inhibits Notch signaling (Extended Data Fig. 8c) ${ }^{9}$. Induction of MAML-Cre mice 10 days post-DEN treatment accelerated tumor removal, proportionally to the expansion of DN-Maml1 mutant clones (Fig. 4b-d and Extended Data Fig. 8d-g). Analysis of images of tumors surrounded by DNMaml1 clones suggest a mechanism of extrusion by competition, similar to that described in the skin and intestinal epithelium (Fig. 4e and Extended Data Fig. 9a-c) ${ }^{17-19}$. The loss of tumors in these experiments cannot be explained by a model that does not include the effects of the surrounding mutant clones (Supplementary information). Instead, the results are compatible with a model where tumors are eliminated by competition with highly fit clones in the adjacent normal epithelium (Supplementary information). To challenge this hypothesis we explored the effect of neutralizing clonal competition following tumor formation. We have previously shown that Notch1 inactivating mutations confer the strongest competitive advantage in the DEN-treated EE ${ }^{11}$. Therefore, inactivating Notch signaling by treating mice with Dibenzazepine (DBZ) would be expected to 'level up' the competitive fitness across the tissue and decrease tumor loss, as mutant epithelial clones would have no advantage over tumors ${ }^{9}$ (Extended Data Fig. 10a-b and Supplementary information). The results show that DBZ treatment does indeed decrease tumor loss (Extended Data Fig. 10c-d). We conclude that expansion of mutant clones in the normal EE 
removes early neoplasms through competition, acting as a selective pressure for tumor survival (Supplementary information).

\section{Discussion}

Our bodies are protected with highly efficient innate mechanisms to suppress tumors, typically exemplified by the immune system. In this study, we have identified a tumorprotective mechanism, independent of the immune system, by which expanding highly fit mutant clones present in normal EE outcompete and eliminate early neoplastic lesions. Importantly, the results imply that the survival of early neoplasms does not depend solely on the mutations they carry, but also on the mutational landscape of the neighboring normal tissue. These findings help reconcile the seemingly conflicting observations of a relatively low cancer incidence despite the large burden of cancer-associated mutations observed in healthy tissues ${ }^{1-7}$, and suggest that some mutations found in normal tissues may contribute to the maintenance of homeostasis, with clear implications in cancer and aging.

Whether an analogous anti-tumor mechanism exists in humans is unknown. However, the existence of small neoplasms phenotypically similar to those found in our mutagenized mouse model, in otherwise healthy human EE (Extended Data Fig. 10e-f), together with the observed preferential colonization of Notch1-mutant clones in human normal EE versus squamous neoplasms ${ }^{2,5}$, suggest the anti-tumor mechanisms described here may be common across species. Likewise, the high burden of cancer-driver somatic mutations identified in other normal human epithelia including that from skin, endometrium, lung and colon, suggests a similar mechanism of tumor purging by competition with mutant clones in adjacent normal epithelium could also take place in these tissues ${ }^{1-7}$.

Our results show that modifying the relative competitive fitness of clones in normal epithelium and neoplastic lesions influences early tumor dynamics. Future studies are needed to explore whether environmental factors that alter the mutational landscape of normal tissues also impact early tumorigenesis through the mechanism described here. This study further suggests that treatments aimed at manipulating the mutant clonal 
architecture of normal epithelium might be exploited as an early cancer prevention strategy 10,12

\section{References}

1 Lee-Six, H. et al. The landscape of somatic mutation in normal colorectal epithelial cells. Nature 574, 532-537, doi:10.1038/s41586-019-1672-7 (2019).

2 Martincorena, I. et al. Somatic mutant clones colonize the human esophagus with age. Science 362, 911-917, doi:10.1126/science.aau3879 (2018).

3 Martincorena, l. et al. Tumor evolution. High burden and pervasive positive selection of somatic mutations in normal human skin. Science 348, 880-886, doi:10.1126/science.aaa6806 (2015).

4 Suda, K. et al. Clonal Expansion and Diversification of Cancer-Associated Mutations in Endometriosis and Normal Endometrium. Cell reports 24, 1777-1789, doi:10.1016/j.celrep.2018.07.037 (2018).

5 Yokoyama, A. et al. Age-related remodelling of oesophageal epithelia by mutated cancer drivers. Nature 565, 312-317, doi:10.1038/s41586-018-0811-x (2019).

6 Moore, L. et al. The mutational landscape of normal human endometrial epithelium. Nature 580, 640-646, doi:10.1038/s41586-020-2214-z (2020).

7 Yoshida, K. et al. Tobacco smoking and somatic mutations in human bronchial epithelium. Nature 578, 266-272, doi:10.1038/s41586-020-1961-1 (2020).

8 Vermeulen, L. et al. Defining stem cell dynamics in models of intestinal tumor initiation. Science 342, 995-998, doi:10.1126/science.1243148 (2013).

9 Alcolea, M. P. et al. Differentiation imbalance in single oesophageal progenitor cells causes clonal immortalization and field change. Nature cell biology 16, 615-622, doi:10.1038/ncb2963 (2014).

10 Fernandez-Antoran, D. et al. Outcompeting p53-Mutant Cells in the Normal Esophagus by Redox Manipulation. Cell stem cell 25, $329-341$ e326, doi:10.1016/j.stem.2019.06.011 (2019).

11 Colom, B. et al. Spatial competition shapes the dynamic mutational landscape of normal esophageal epithelium. Nature genetics 52, 604-614, doi:10.1038/s41588020-0624-3 (2020).

12 Murai, K. et al. Epidermal Tissue Adapts to Restrain Progenitors Carrying Clonal p53 Mutations. Cell stem cell, doi:10.1016/j.stem.2018.08.017 (2018).

13 Doupe, D. P. et al. A single progenitor population switches behavior to maintain and repair esophageal epithelium. Science 337, 1091-1093, doi:10.1126/science.1218835 (2012).

14 Piedrafita, G. et al. A single-progenitor model as the unifying paradigm of epidermal and esophageal epithelial maintenance in mice. Nature communications 11, 1429, doi:10.1038/s41467-020-15258-0 (2020). 
15 Frede, J., Greulich, P., Nagy, T., Simons, B. D. \& Jones, P. H. A single dividing cell population with imbalanced fate drives oesophageal tumour growth. Nature cell biology 18, 967-978, doi:10.1038/ncb3400 (2016).

16 Martincorena, l. et al. Universal Patterns of Selection in Cancer and Somatic Tissues. Cell 171, 1029-1041 e1021, doi:10.1016/j.cell.2017.09.042 (2017).

17 Brown, S. et al. Correction of aberrant growth preserves tissue homeostasis. Nature 548, 334-337, doi:10.1038/nature23304 (2017).

18 Ellis, S. J. et al. Distinct modes of cell competition shape mammalian tissue morphogenesis. Nature 569, 497-502, doi:10.1038/s41586-019-1199-y (2019).

19 Kon, S. et al. Cell competition with normal epithelial cells promotes apical extrusion of transformed cells through metabolic changes. Nature cell biology 19, 530-541, doi:10.1038/ncb3509 (2017).

20 Sakuntabhai, A. et al. Mutations in ATP2A2, encoding a Ca2+ pump, cause Darier disease. Nature genetics 21, 271-277, doi:10.1038/6784 (1999).

21 Liu, L. H., Boivin, G. P., Prasad, V., Periasamy, M. \& Shull, G. E. Squamous cell tumors in mice heterozygous for a null allele of Atp2a2, encoding the sarco(endo)plasmic reticulum Ca2+-ATPase isoform $2 \mathrm{Ca} 2+$ pump. The Journal of biological chemistry 276, 26737-26740, doi:10.1074/jbc.C100275200 (2001).

22 Shultz, L. D. et al. Human lymphoid and myeloid cell development in NOD/LtSz-scid IL2R gamma null mice engrafted with mobilized human hemopoietic stem cells. Journal of immunology 174, 6477-6489, doi:10.4049/jimmunol.174.10.6477 (2005).

$23 \mathrm{Li}, \mathrm{H}$. Aligning sequence reads, clone sequences and assembly contigs with BWAMEM. ArXiv, 1303.3997 (2013).

24 Gerstung, M., Papaemmanuil, E. \& Campbell, P. J. Subclonal variant calling with multiple samples and prior knowledge. Bioinformatics 30, 1198-1204, doi:10.1093/bioinformatics/btt750 (2014).

25 Alexandrov, L. et al. (bioRxiv, 2018).

26 Rosenthal, R., McGranahan, N., Herrero, J., Taylor, B. S. \& Swanton, C. DeconstructSigs: delineating mutational processes in single tumors distinguishes DNA repair deficiencies and patterns of carcinoma evolution. Genome biology 17, 31, doi:10.1186/s13059-016-0893-4 (2016).

27 Jones, D. et al. cgpCaVEManWrapper: Simple Execution of CaVEMan in Order to Detect Somatic Single Nucleotide Variants in NGS Data. Current Protocols in Bioinformatics 56, 15.10.11-15.10.18, doi:10.1002/cpbi.20 (2016).

28 Menzies, A. et al. VAGrENT: Variation Annotation Generator. Current Protocols in Bioinformatics 52, 15.18.11-15.18.11, doi:10.1002/0471250953.bi1508s52 (2015).

29 Scheinin, I. et al. DNA copy number analysis of fresh and formalin-fixed specimens by shallow whole-genome sequencing with identification and exclusion of problematic regions in the genome assembly. Genome research 24, 2022-2032, doi:10.1101/gr.175141.114 (2014).

30 Kuilman, T. et al. CopywriteR: DNA copy number detection from off-target sequence data. Genome biology 16, 49, doi:10.1186/s13059-015-0617-1 (2015).

31 Nik-Zainal, S. et al. Mutational processes molding the genomes of 21 breast cancers. Cell 149, 979-993, doi:10.1016/j.cell.2012.04.024 (2012).

32 Tumbar, T. et al. Defining the epithelial stem cell niche in skin. Science 303, 359-363 (2004). 
33 Toni, T. \& Stumpf, M. P. Simulation-based model selection for dynamical systems in systems and population biology. Bioinformatics 26, 104-110, doi:10.1093/bioinformatics/btp619 (2010).

34 Klinger, E., Rickert, D. \& Hasenauer, J. pyABC: distributed, likelihood-free inference. Bioinformatics 34, 3591-3593, doi:10.1093/bioinformatics/bty361 (2018).

35 Consortium, T. Integrated genomic characterization of oesophageal carcinoma. Nature 541, 169-175, doi:10.1038/nature20805 (2017).

36 Klein, A. M., Brash, D. E., Jones, P. H. \& Simons, B. D. Stochastic fate of $<\mathrm{em}>\mathrm{p} 53-$ $</ e m>$ mutant epidermal progenitor cells is tilted toward proliferation by UV B during preneoplasia. Proceedings of the National Academy of Sciences 107, 270-275, doi:10.1073/pnas.0909738107 (2010).

37 Clayton, E. et al. A single type of progenitor cell maintains normal epidermis. Nature 446, 185-189 (2007).

38 Bailey, N. T. J. The Elements of Stochastic Processes with Applications to the Natural Sciences. (Wiley, 1964).

39 Orr, H. A. THE POPULATION GENETICS OF ADAPTATION: THE DISTRIBUTION OF FACTORS FIXED DURING ADAPTIVE EVOLUTION. Evolution 52, 935-949, doi:10.1111/j.1558-5646.1998.tb01823.x (1998).

40 Greaves, M. \& Maley, C. C. Clonal evolution in cancer. Nature 481, 306-313, doi:nature10762 [pii] 10.1038/nature10762 (2012).

41 Hall, M. W. J., Jones, P. H. \& Hall, B. A. Relating evolutionary selection and mutant clonal dynamics in normal epithelia. Journal of the Royal Society Interface 16, 20190230, doi:10.1101/480756 (2019). 


\section{Figures}

a

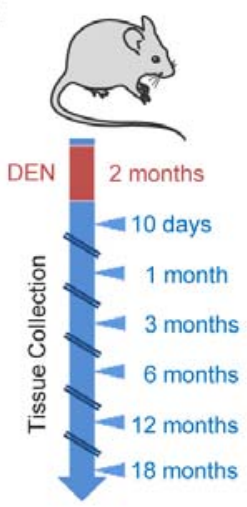

b

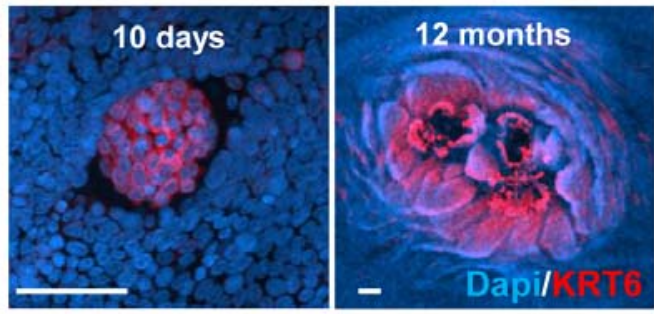

C

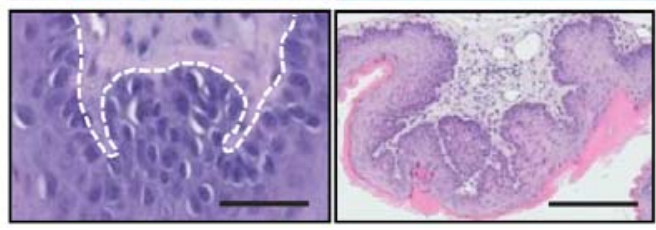

d

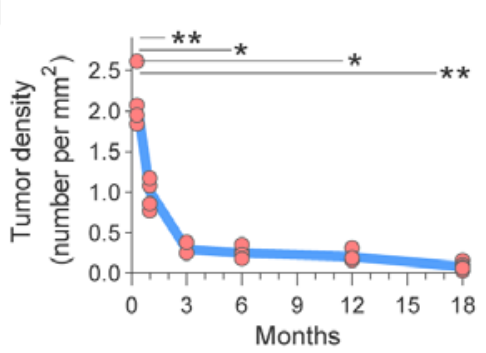

e
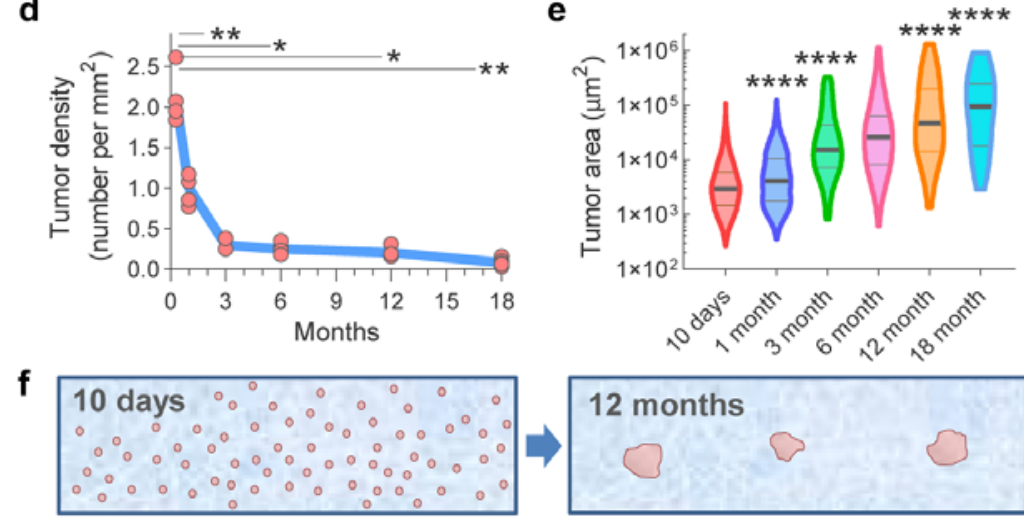

12 months

Fig. 1. Most esophageal tumors are lost. (a) Wild-type mice received DEN in drinking water. The esophagus was collected at the indicated time-points. (b-c) Confocal (b) and H\&E (c) images of EE tumors from mice collected 10-days or 12-months post-DEN treatment. Scalebars: $50 \mu \mathrm{m}$. Tissues in (b) were stained for Dapi (blue) and Keratin 6 (KRT6, red) to identify tumors. White-dots in (c) delineate the basal cell layer. (d-e) Density (d) and size (e) of tumors at the indicated time-points post-DEN treatment. Blue line in (d) connects the Mean values (Two-sided Mann-Whitney test, vs 10-days). Violin plot lines in (e) show median and quartiles (Two-sided Mann-Whitney test, vs 10-days). Number of mice (and tumors) are: $10 \mathrm{~d}=4(588), 1 \mathrm{~m}=5(408), 3 \mathrm{~m}=3(73), 6 \mathrm{~m}=5(92), 12 \mathrm{~m}=4(58)$ and $18 \mathrm{~m}=6(42)$. (f) Cartoon illustrating tissue tumor clearance over time. ${ }^{*} p<0.05, * * p<0.01, * * * * p<0.0001$. 


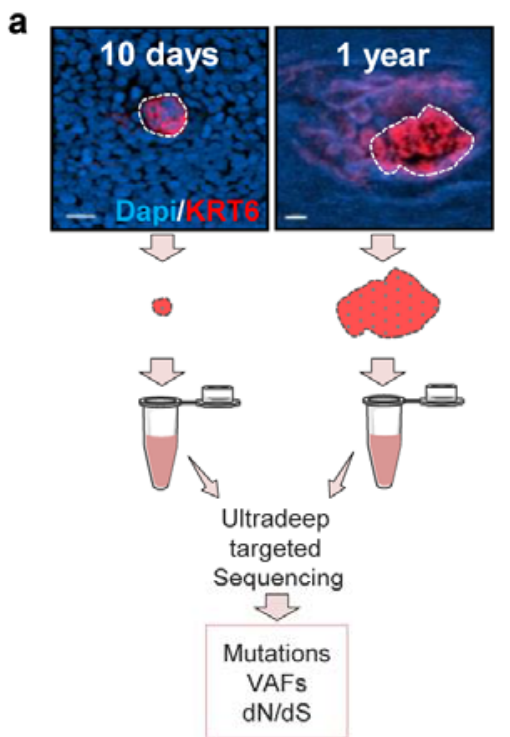

c $\quad \mathrm{dN} / \mathrm{dS}$ ratios $(\mathrm{q}<0.05)$
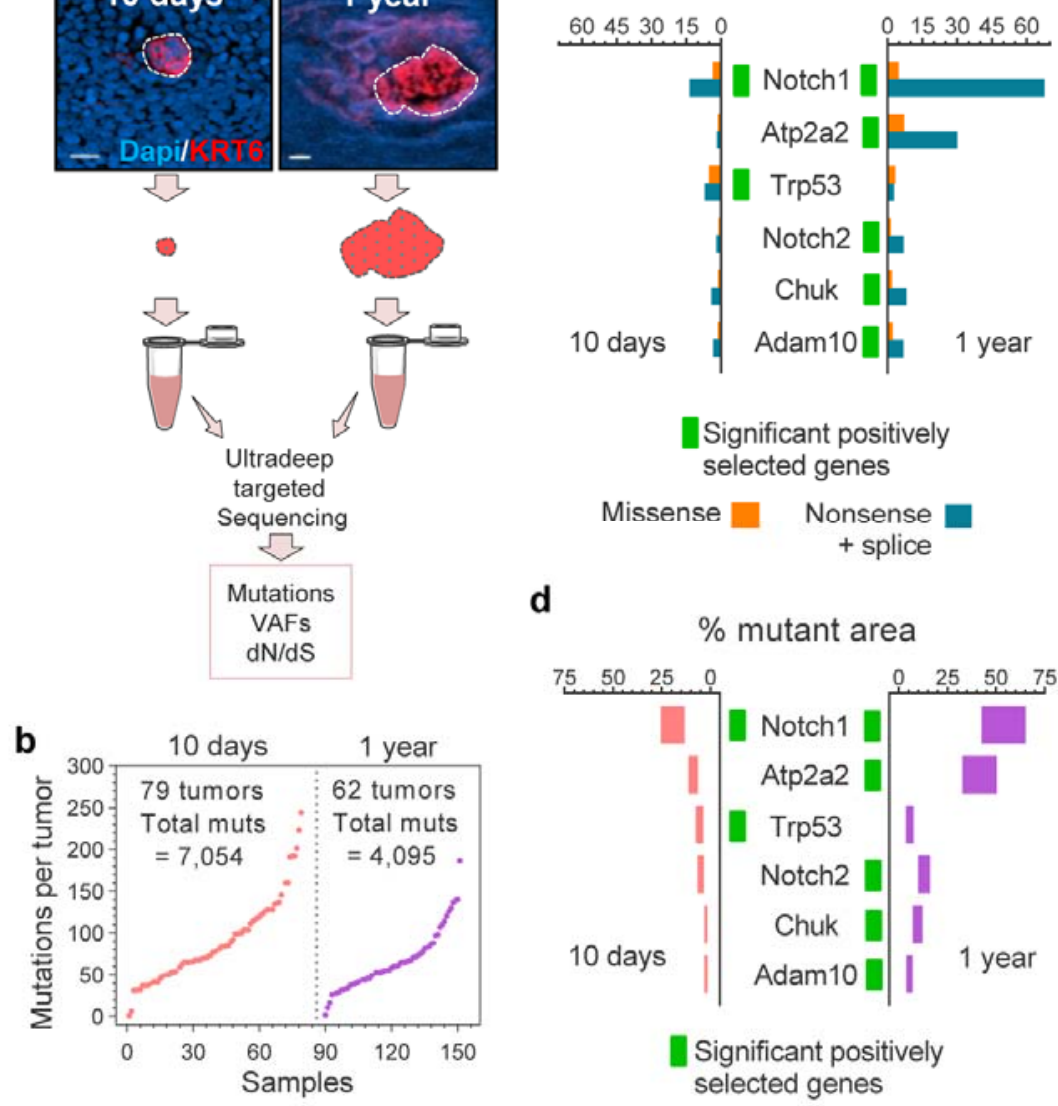

d

$\%$ mutant area

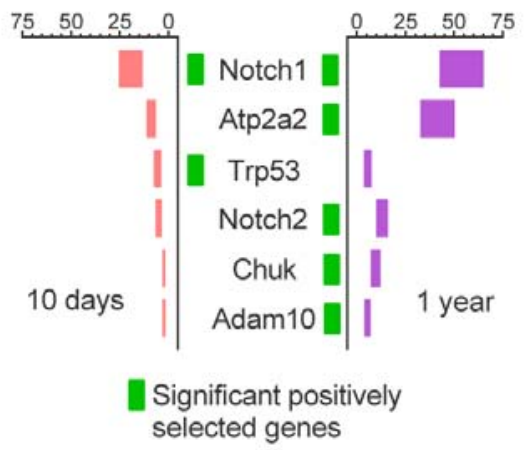

Fig. 2. Sequencing of newly born and surviving tumors reveals patterns of selection. (a) $E E$ tumors were collected at 10-days and 12-months post-DEN treatment as in Figure 1, and ultra-deep targeted sequenced using a 192 gene bait-set. Scale-bars: 50 $\mu \mathrm{m}$. (b) Number of mutations per tumor, including essential splice, frameshift, missense, nonsense and silent mutations. (c) dN/dS ratios for missense and truncating (nonsense + essential splice site) substitutions indicating genes under significant positive selection in the tumors (green rectangles, $q<0.05$ calculated with $\mathrm{R}$ package $\mathrm{dNdSCv}{ }^{16}$ ). (d) Estimated percentage of tumor area carrying non-synonymous mutations for each gene. Range indicates upper and lower bound estimates. Green rectangles indicate $\mathrm{dN} / \mathrm{dS}$ positively selected genes for each timepoint. 


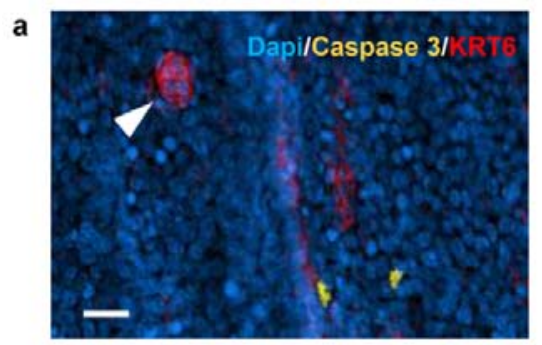

b
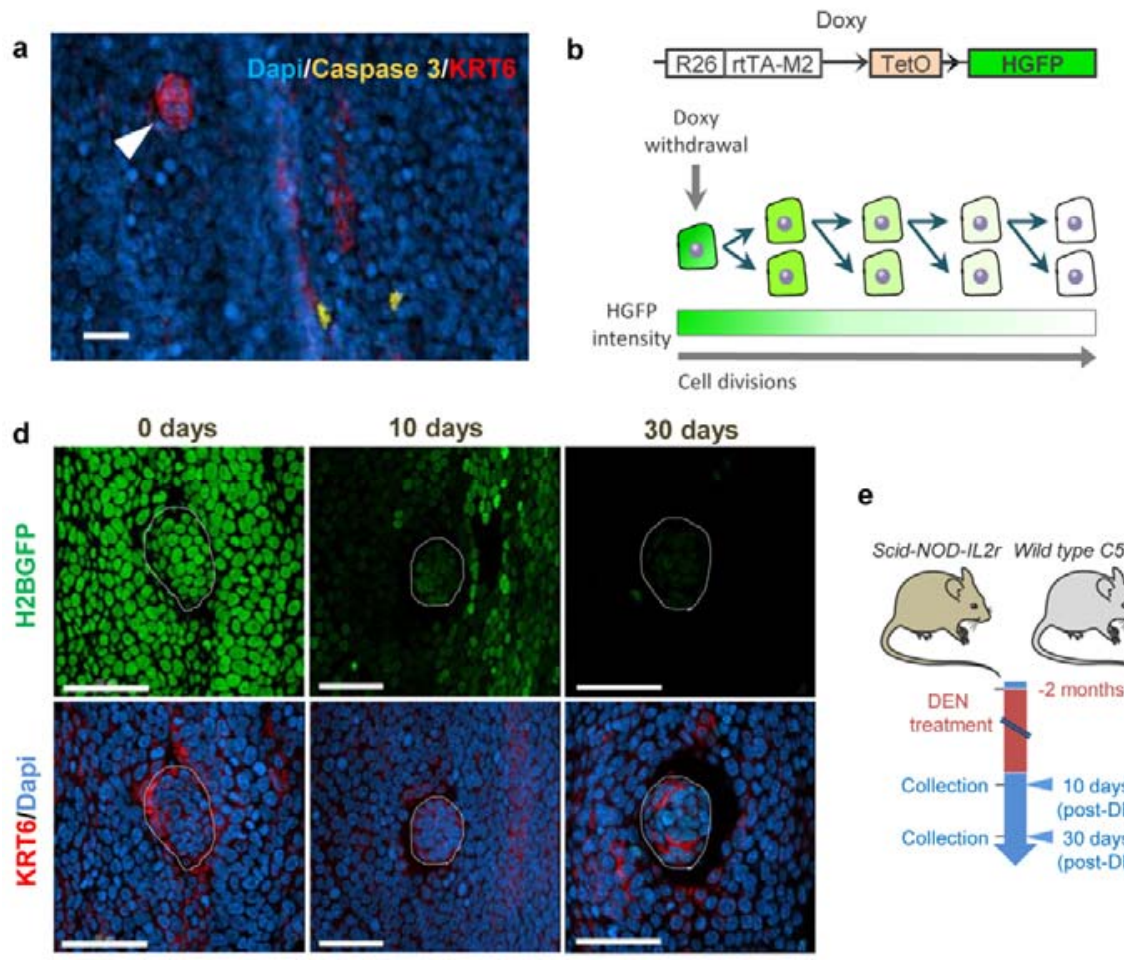

c
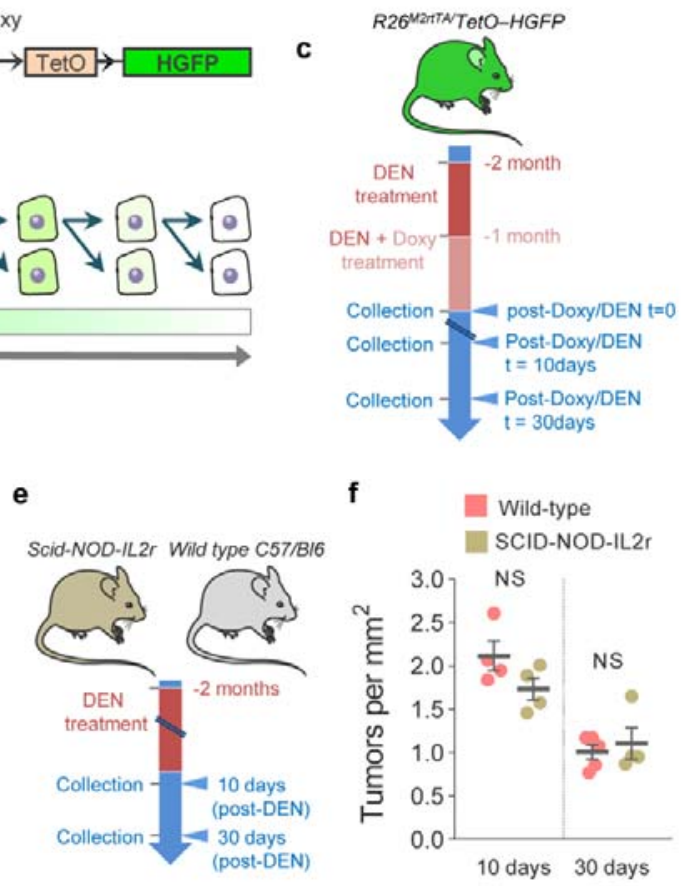

Fig. 3. Early tumors are not eliminated by tumor cell apoptosis, abnormal proliferation or the immune system. (a) Representative confocal image of a 10-day post-DEN EE immunostained for activated Caspase $3^{+}$and KRT6. The image shows two apoptotic cells in the normal epithelium (yellow). No apoptotic cells were detected in the tumors (arrowhead) (0/23 tumors analyzed from 2 mice). Scale-bar: $20 \mu \mathrm{m}$. (b) In vivo label-retaining assay using Rosa $26^{\text {M2rtA }} /$ TetO-HGFP transgenic mice to measure the rate of progenitor cell division. In this strain, expression of a stable Histone-Green Fluorescent Protein (HGFP) is activated by treatment with Doxycycline (Doxy). When Doxy is withdrawn, levels of the stable protein fall at a rate dependent on the rate of cell division. (c) Protocol: Rosa26 $6^{\text {M2rtTA } / T e t O-H G F P ~ m i c e ~}$ received DEN for one month followed by DEN + Doxy for another month. Tissues were collected at times 0,10 and 30 days after Doxy/DEN withdrawal. (d) Confocal images showing HGFP expression in tumors (dotted lines) and their normal adjacent EE at different time-points post-Doxy withdrawal. Scale bars: $50 \mu \mathrm{m}$. (e) Protocol: Wild-type and immunocompromised Scid-NOD-IL2r mice were treated with DEN for two months and the tissues collected 10 or 30 days after DEN withdrawal. (f) Number of tumors per $\mathrm{mm}^{2}$ of EE in wild-type and Scid-NOD-IL2r mice, collected 10-days $(n=4)$ or 30-days ( $n=5$ and 4, respectively) post-DEN treatment. Mean \pm s.e.m. NS= not significant (Two-sided MannWhitney test). 
a
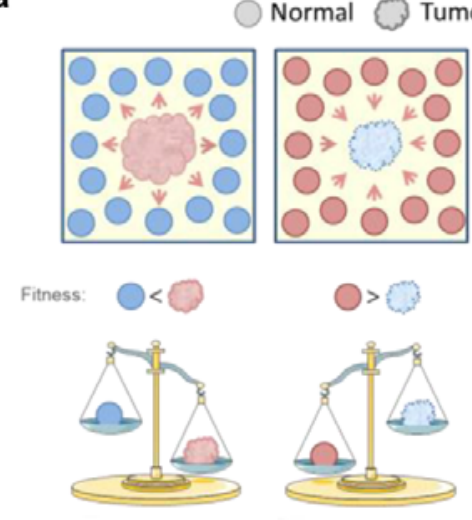

Tumour growth

d
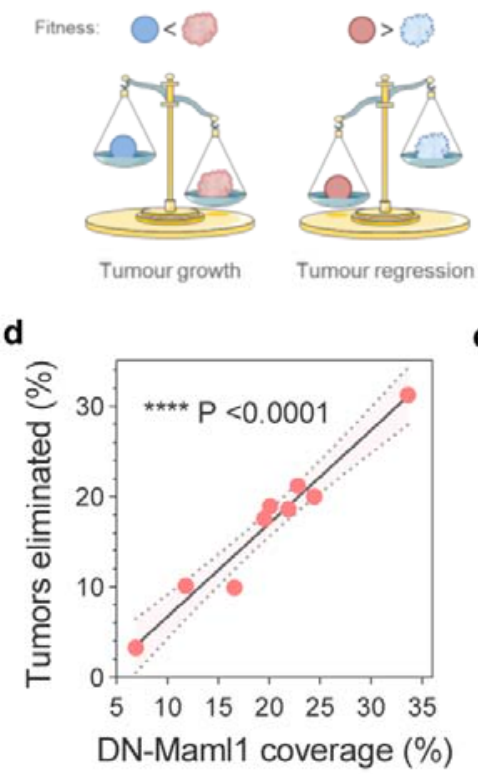

b
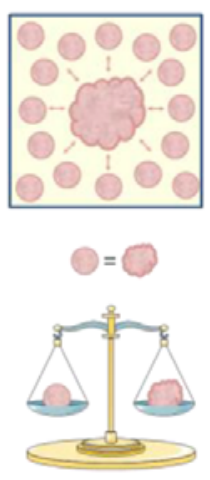

Neutral drift

e

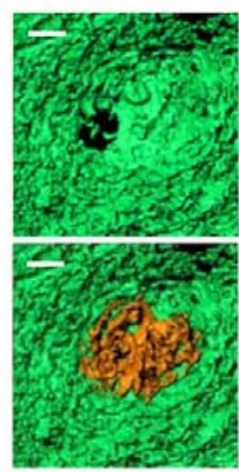

c
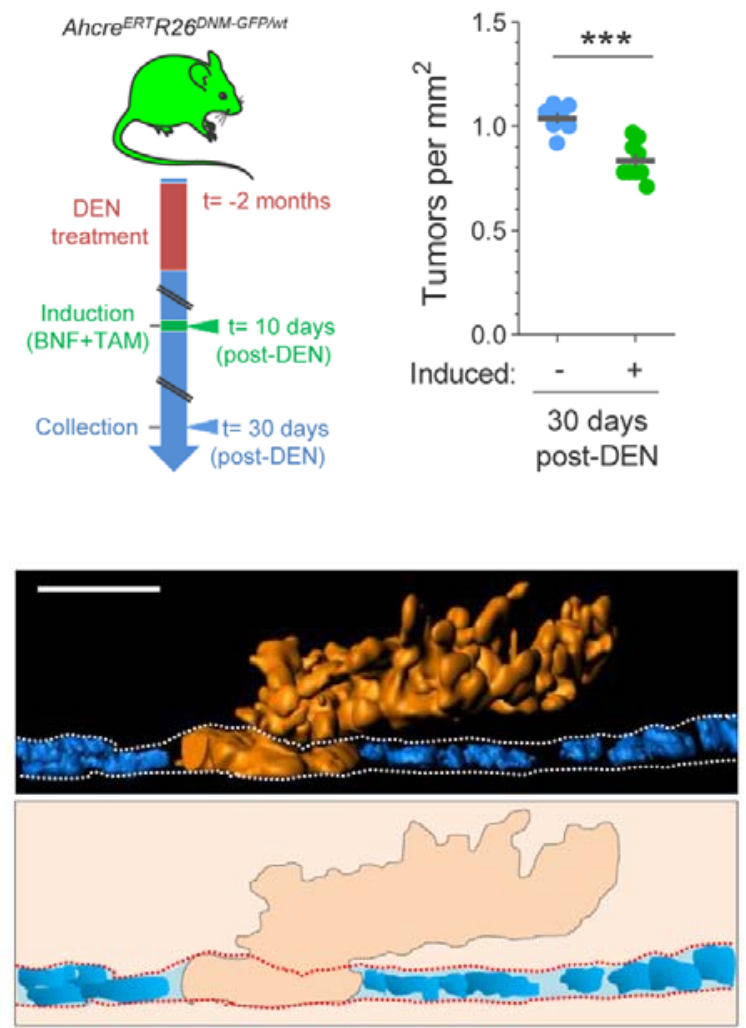

Fig. 4. Elimination of early tumors by expansion of mutant clones in the adjacent normal epithelium. (a) In our model the survival of tumors is dependent on their relative competitive fitness compared to the surrounding normal tissue. This predicts 3 possible scenarios: 1) tumor cells are fitter than those in the normal adjacent epithelium (left), resulting in tumor growth; 2) Tumor cells are less fit than the adjacent normal (middle), resulting in tumor regression and elimination, and 3) cells in tumor and adjacent normal epithelium are equally fit (right), resulting in neutral competition. (b) Protocol: Ahcre ${ }^{E R T}$ Rosa26 ${ }^{\text {wt/DNM-GFP }}$ mice (Extended Data Fig. 10b) received DEN for two months. 10 days after DEN withdrawal DN-Maml1 clones were induced and tissues harvested twenty days later. Control mice received DEN but were not induced. (c) Number of tumors per $\mathrm{mm}^{2}$ of EE in Ahcre ${ }^{E R T}$ Rosa26 $6^{\text {wt/DNM-GFP }}$ induced and non-induced controls, as in (b). (d) Correlation between the area covered by DN-Mam1 clones and tumors being eliminated from the EE. Each dot represents a mouse $(n=9)$. Line shows the Pearson correlation $\left(R^{2}=0.9541\right)$ with 95\% confidence interval (colored area). (e) Top view (left), lateral view (right top) and schematic (right bottom) of a tumor (orange) surrounded by a DN-Maml1 clone (green). Dapi is shown in blue. Scale-bars: $20 \mu \mathrm{m}$. 


\section{Materials and Methods}

Mice. All animal experiments were conducted at the MRC ARES facility, Babraham or the RSF facility at the Wellcome Sanger Institute according to the UK Home Office Project Licenses 70/7543, P14FED054 or PF4639B40. Mice were housed in individually ventilated cages and fed on standard chow. Male and female adult mice were used for experiments.

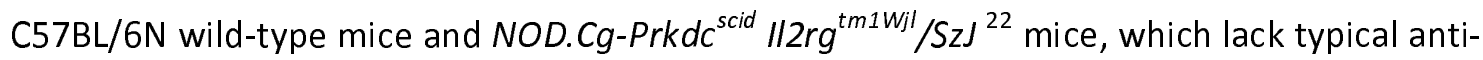
tumor immune responses including a complete absence of $B$ and $T$ lymphocytes, NK cells, and defective dendritic cells and macrophages, were obtained from core colonies bred at the RSF facility. Double mutant $R 26^{M 2 r t T A} /$ TetO-HGFP and AhCre ${ }^{E R T} R o s a 26^{f I D N M-G F P / w t}$ strains on a $\mathrm{C} 57 \mathrm{BL} / 6 \mathrm{~N}$ background were generated as described previously $9,13,15$.

Chemical model of mouse esophageal neoplasia. Adult ( $>10$ week old) mice were treated with Diethylnitrosamine (DEN) in sweetened drinking water (40?mg per 1,000? $\mathrm{ml}$ ) for twenty-four hours, three days a week (Monday, Wednesday and Fridays) for eight weeks 9,15. Animals received sweetened water in between DEN dosages and normal water after DEN treatment was completed. Tissue (esophagus) samples were collected from 10 days to 18 months after DEN treatment.

Mouse whole-esophagus sampling and processing. To prepare the esophagus for wholetissue staining and imaging, tissues were dissected and placed in cold PBS. The esophageal tube was cut longitudinally and the muscle layer removed by pulling gently with microforceps. To separate the epithelium from the underlying submucosa the entire tissue was incubated for 2-3 hours in $5 \mathrm{mM}$ EDTA at $37^{\circ} \mathrm{C}$ and both layers were pulled away under a dissecting microscope with fine forceps. The whole esophageal epithelium was then flattened, fixed in $4 \%$ paraformaldehyde for $30 \mathrm{~min}$ at room temperature, washed in PBS and finally stored at $4{ }^{\circ} \mathrm{C}$ until further processing.

Mouse whole-esophagus immunostaining. For immunostaining, whole-tissue esophageal epitheliums were blocked for one hour in $800 \mu \mathrm{l}$ of staining buffer $10.5 \%$ bovine serum albumin, $0.25 \%$ fish skin gelatin, $0.5 \%$ Triton X-100 in PBS and $10 \%$ donkey serum) followed by an overnight incubation at room temperature with primary antibodies against KRT6, GFP, CD31, CD45 or Caspase 3, in staining buffer. Samples were then washed four times $(x 20$ minutes) with $0.2 \%$ Tween-20 in PBS and, when necessary, incubated with secondary antibodies in staining buffer for three hours at room temperature. Samples were finally washed as above, incubated overnight at room temperature with $1 \mu \mathrm{g} / \mathrm{ml}$ DAPI or $0.4 \mu \mathrm{M}$ TO-PRO ${ }^{\text {TM }}-3$ Iodide solution to stain cell nuclei, and mounted using VECTASHIELD Mounting Media before image acquisition. 
Human samples. Esophagi were obtained from deceased organ donors. Informed consent for the use of tissue was obtained from the donor's family (REC reference: 15/EE/0152 NRES Committee East of England - Cambridge South). A full thickness segment of mid-esophagus was excised within 60 minutes of circulatory arrest and preserved in University of Wisconsin (UW) organ preservation solution (Belzer UW ${ }^{\circledR}$ Cold Storage Solution, Bridge to Life, USA) at $4{ }^{\circ} \mathrm{C}$ until further processing.

Immunostaining of human esophagus. Donor esophageal samples were cut longitudinally, flattened and the muscle layer removed. Samples were blocked for one hour in $1 \mathrm{~mL}$ of staining buffer $(0.5 \%$ bovine serum albumin, $0.25 \%$ fish skin gelatin, $1 \%$ Triton $X-100$ and $10 \%$ donkey serum in PHEM buffer (60 mM PIPES, 25 mM HEPES, 10 mM EGTA, and 4mM $\mathrm{MgSO} 4 \cdot 7 \mathrm{H} 20)$ ), followed by two days incubation at room temperature with primary antibody against KRT6 in staining buffer. Samples were then washed for 2 days in PHEM buffer before incubation with secondary antibody in staining buffer for two days at room temperature. Samples were washed as above and incubated overnight at room temperature with $0.4 \mu \mathrm{M}$ TO-PRO ${ }^{\text {TM }}-3$ Iodide solution to stain cell nuclei. Finally, samples were incubated for one hour at $37^{\circ} \mathrm{C}$ and then mounted, both in RapiClear 1.52 (SUNJin lab) solution, for image acquisition.

Confocal microscopy. To obtain high quality confocal images of the entire mouse esophagus, whole-mounted tissues were imaged in a Leica TCS SP8 confocal microscope coupled to a high precision motorized stage. Contiguous 3D images of all epithelial layers (basal + suprabasal) were obtained and merged computationally using the mosaic function of the Leica Software. Typical settings for acquisition of multiple z stacks were $2.5 \mu \mathrm{m}$ z-step size, zoom $x 1$, optimal pinhole, line average 4, scan speed 400 ? $\mathrm{Hz}$ and a resolution of 1,024 $\times 1,024$ pixels using a $10 \mathrm{X}$ HC PL Apo CS Dry objective with a 0.4NA. Visualization and image analysis was performed using IMARIS (bitplane), ImageJ or Volocity 3D Image Analysis Software (Perkin Elmer). Otherwise, close up images were obtained with $\times 10, \times 20$ or $\times 40$ objectives with typical settings for acquisition of $z$ stacks being optimal pinhole, line average $3-4$, scan speed $400-600$ ? $\mathrm{Hz}$ and a resolution of $512 \times 512$ or $1,024 \times 1,024$ pixels.

KRT6 staining as an early marker of mouse esophageal epithelial tumors. KRT6 is a keratinocyte stress-induced protein upregulated at both RNA and protein levels in mouse esophageal tumors ${ }^{15}$. Here we used whole-tissue KRT6 staining to identify DEN-generated neoplasms in the esophageal epithelium from as early as 10 days post-DEN treatment. To characterize the protein expression levels in the tumors relative to the surrounding normal epithelium, the KRT6 staining profile in and around tumors was analyzed from esophagi collected 10 days after DEN treatment. The intensity of KRT6 and the nuclear marker DAPI were measured with ImageJ from defined ROls containing tumors or their normal surrounding epithelia. 
Tissue histology. Esophagus from DEN-treated mice were dissected, fixed in $10 \%$ formalin for at least 24 hours and stored at $4{ }^{\circ} \mathrm{C}$. Tissues were then embedded in paraffin and cut at 5 $\mu \mathrm{m}$ thickness. Sections were stained with hematoxylin and eosin and scanned.

Quantification of tumor number and size. To quantify the number and the area of the neoplasms present in the esophagus of carcinogen-treated mice at different time points, whole-tissues were stained with antibodies against KRT6 and a nuclear marker such as DAPI or TO-PRO ${ }^{\text {TM }}$-3. High definition 3D confocal images of the whole esophagus were obtained and visualized with IMARIS software and ImageJ. Tumors were manually identified by shape (based on the nuclear staining) and KRT6 staining profile. The number of tumors quantified in the entire esophagus was divided by the total area of the tissue to obtain the number of tumors per $\mathrm{mm}^{2}$ of esophageal epithelium. The size of individual neoplasms was calculated by assuming an ellipse shape using the following formulae: $A=\left(D_{1} D_{2} \pi\right) / 2$, where $D_{1}$ and $D_{2}$ are the length of the major and minor diameters measured with IMARIS software.

\section{Targeted sequencing of 10 days and 1 year esophageal neoplasms}

Tissue preparation. Mice were treated with DEN for eight weeks and culled 10 days or 1 year after treatment. Mouse esophagi were dissected, cut longitudinally and the muscle layer removed. Tissues were incubated for $2-3$ hours in $5 \mathrm{mM}$ EDTA at $37{ }^{\circ} \mathrm{C}$ to separate the epithelium from the underlying submucosa. The whole epithelium was flattened, fixed in $4 \%$ paraformaldehyde for $30 \mathrm{~min}$ at room temperature and washed in PBS. Tissues were then immuno-stained for KRT6 and the nuclei labelled with DAPI or TO-PRO ${ }^{\text {TM }}-3$ to identify the tumors before being imaged by confocal microscopy as described above.

Excision and digestion of neoplasms. Esophageal neoplasms from animals culled 10 days or 1 year post-DEN treatment were identified from the confocal images and manually cut using micro-scalpels under a fluorescent dissecting microscope. Tumors were placed in $0.2 \mathrm{ml}$ collector tubes containing $20 \mu$ lysis buffer ( $30 \mathrm{mM}$ Tris- $\mathrm{HCl} \mathrm{pH}=8.0,0.5 \%$ Tween-20, $0.5 \%$ NP-40 and $0.056 \mu \mathrm{AU}$ Proteinase $\mathrm{K}$ ) and kept on ice until digested at $56{ }^{\circ} \mathrm{C}$ for 60 minutes followed by $76^{\circ} \mathrm{C}$ for 30 minutes, before being finally stored at $-80^{\circ} \mathrm{C}$. For each animal, one or more pieces of ear skin were cut and processed as above to be used as germline controls.

Library preparation. Samples were processed using a recently developed enzymatic fragmentation-based library preparation method specifically designed to detect small amounts of DNA (low-input) without the need for whole genome amplification ${ }^{1}$. Briefly, genomic DNA was purified by mixing $20 \mu$ of the lysed samples with $50 \mu$ Ampure XP beads (Beckman Coulter), and left for 5 minutes at room temperature. After magnetic separation, 
bead-DNA samples were washed twice with $75 \%$ ethanol and re-suspended in $26 \mu \mathrm{TE}$ buffer (10 mM Tris-HCl, 1 mM EDTA). For DNA fragmentation, samples were mixed with $7 \mu \mathrm{l}$ $5 x$ Ultra II FS buffer and $2 \mu$ U Ultra II FS enzyme (New England BioLabs), and incubated 12 minutes at $37{ }^{\circ} \mathrm{C}$ followed by 30 minutes at $65^{\circ} \mathrm{C}$. Samples were then incubated with a $30 \mu \mathrm{l}$ ligation mix, $1 \mu$ ligation enhancer (New England BioLabs), $0.9 \mu$ l nuclease-free water and $0.1 \mu \mathrm{l}$ duplexed adapter (100 $\mu \mathrm{M}$; 5?-ACACTCTTTCCCTACACGACGCTCTTCCGATC*T-3?], 5? phos-GATCGGAAGAGCGGTTCAGCAGGAATGCCGAG-3]). Adapter-ligated libraries were then purified by mixing with $65 \mu \mathrm{l}$ Ampure XP beads (Beckman Coulter) followed by magnetic separation and elution with $65 \mu \mathrm{l}$ TE buffer. For the PCR reaction, $21.5 \mu \mathrm{l}$ of the DNA samples were mixed with $25 \mu \mathrm{l} \mathrm{KAPA} \mathrm{HiFi} \mathrm{HotStart} \mathrm{ReadyMix} \mathrm{(KAPA} \mathrm{Biosystems),} 1 \mu \mathrm{l} \mathrm{PE1.0}$ primer $(100 \mu \mathrm{M}$;

5? AATGATACGGCGACCACCGAGATCTACACTCTTTCCCTACACGACGCTCTTCCGATC*T-3] and $2.5 \mu \mathrm{l}$ iPCR-Tag (40 $\mu \mathrm{M}$;

5?CAAGCAGAAGACGGCATACGAGATXGAGATCGGTCTCGGCATTCCTGCTGAACCGCTCTTCCGATC3 ?]), in which ' $X$ ' represents one of 96 unique 8-base indexes. $P C R$ conditions were: 5 min at $98 ?^{\circ} \mathrm{C}$, followed by 12 cycles of $30 \mathrm{~s}$ at 98$]^{\circ} \mathrm{C}, 30 \mathrm{~s}$ at 65$]^{\circ} \mathrm{C}$ and $1 \mathrm{~min}$ at 72$]^{\circ} \mathrm{C}$, and finally 5 $\min$ at 72 ? $^{\circ} \mathrm{C}$. Amplified libraries were purified by mixing with Ampure beads at a ratio 0.7:1, adjusted to $2.4 \mathrm{nM}$ with nuclease-free water.

DNA sequencing. A custom bait capture to target the exonic sequences of 192 genes frequently mutated in cancer was designed using Agilent SureDesign. The list of genes selected for ultra-deep targeted sequencing is shown below:

abcb11, abcc2, adam10, adam29, adcy10, aff3, ajuba, akap9, akt1, akt2, apob, arid1a, arid2, arid5b, asx11, atm, atp2a2, atrx, aurka, b2m, bbs9, bcas3, bcl11b, bcr, braf, brca2, c1s, cacna1d, card11, casp8, ccnd1, cdc16, cdh1, cdkn2a, chuk, clgn, cnot1, cntnap4, cobll1, col12a1, copb2, cr2, crebbp, csmd2, ctcf, cul3, cyld, cyp2b13, dclk1, dclre1a, ddr2, dicer1, $d m \times 12, d n m 2, d n m t 3 a, d s t$, egfr, eif2d, ep300, epha2, erbb2, erbb3, erbb4, ezh2, fat1, fat4, fbn2, fbxo21, fbxw7, fgfr1, fgfr2, fgfr3, flg2, flt3, fn1, gcn1l1, grin2a, grm3, gtf3c5, hist1h2bm, hmon1, hras, huwe 1, hydin, igsf1, insrr, iqgap1, irf6, kcnh5, kdm5b, kdm6a, kdr, keap1, kit, klrc3, kmt2c, kmt2d, kras, krt5, krtap4-9, loxhd1, Irp1, Irp1b, Irp2, Itf, maml1, mcm 7, met, mrgprb4, mtor, myof, nf1, nf2, nfe212, nfkb1, nlrp12, notch1, notch2, notch3, notch4, nras, nsd1, nup214, opn3, pard3, pcdha5, pced1b, pde4dip, peg10, pign, pik3ca, pkhd1, plcb1, prex2, psme4, ptch1, pten, ptprt, rac1, rasa1, rb1, rbm46, rhbdf2, ripk2, ripk4, ros1, rpgrip1, rpl10, ryr2, sall1, scn10a, scn11a, scn1a, scn3a, setd2, setx, sgk3, sis, slc13a1, smad4, smarca4, smo, snx25, soat2, sox2, spen, st18, sufu, synm, taf2, tas2r102, tet2, tnr, trp53, trp63, trrap, tsc1, ttc27, usp24, usp26, usp9x, vhl, vmn2r81, vps13b, wnk1, zan, zfhx3, zfp39, zfp457, zfp521, zfp644 and zfp750. 
Samples were multiplexed and sequenced on the HiSeq 2500 platform (Illumina) according to the manufacturer's instructions, with the exception that we used iPCR-Tag (5?AAGAGCGGTTCAGCAGGAATGCCGAGACCGATCTC-3]) to read the library index. Paired-end reads were aligned with BWA-MEM (v0.7.17, https://github.com/lh3/bwa) ${ }^{23}$ with optical and PCR duplicates marked using Biobambam2 (v2.0.86, https://gitlab.com/german.tischler/biobambam2, https://www.sanger.ac.uk/science/tools/biobambam).

Mutation calling with deepSNV. In order to identify somatic mutations present in a small fraction of cells within the samples, we used the ShearwaterML algorithm from the deepSNV package (v1.21.3, https://github.com/gerstung-lab/deepSNV) to call for mutational events on ultra-deep targeted data ${ }^{2,3,24}$. Instead of using a single-matched normal sample, deepSNV uses a collection of deeply-sequenced normal samples as the reference for variant calling that enables the identification of mutations at very low allele frequencies. For this study we used a total of 19 normal samples from 12 animals, providing a normal panel with a combined coverage of 7439x. A depth filter of 50x was used in the final variant call results.

Mutation burden. The average number of mutations per $\mathrm{Mb}$ in a given sample was estimated from the VAFs and the number of bases within the bait set with synonymous mutations (dubbed as synonymous footprint), as described before ${ }^{2,3}$, and assuming no alterations in copy number as indicated by either the whole exome and genome sequencing data (Extended Data Fig. 6). Only the synonymous mutations were used for this calculation as the mutation burden can be inflated by the alterations in strongly selected genes when using targeted sequenced data.

Percentage of mutant epithelium. The size of mutant clones within each sample can be calculated by taking into account the area of the biopsy and the fraction of cells carrying a mutation within the sample, as described previously ${ }^{2,3}$. We have determined that DEN treatment does not alter the copy number variation (Extended Data Fig. 6), therefore, the fraction of cells carrying a particular mutation can be estimated as twice the VAF of that mutation. The lower (=VAF) and upper (=2xVAF) bound estimates of the percentage of tissue covered by clones carrying non-synonymous mutations in a given gene was calculated for each sample ${ }^{2,3}$. The fraction of epithelium covered by the mutant genes was then calculated from the mean of summed VAF (capped at 1.0) of all the samples.

Mutational spectra and signature analysis. Mutational spectra for single base substitutions were plotted and compared to 65 known mutational signatures present in human cancers ${ }^{25}$ using linear decomposition with the deconstructSigs $R$ package (https://github.com/raerose01/deconstructSigs) ${ }^{26}$. The mutational spectra across DEN- 
treated samples were highly consistent, precluding deconvolution into separate signatures (either known or de novo).

Gene selection (dN/dS). We used the maximum-likelihood implementation of the $\mathrm{dNdScv}$ algorithm (v0.0.1.0, https://github.com/im3sanger/dndscv) to identify genes under positive selection ${ }^{16}$. $\mathrm{dNdSCv}$ estimates the ratio of non-synonymous to synonymous mutations across genes, controlling for the sequence composition of the gene and the mutational signatures, using trinucleotide context-dependent substitution matrices to avoid common mutation biases affecting $d N / d S$. Values of $d N / d S$ significantly higher than 1 indicate an excess of non-synonymous mutations in that particular gene and therefore imply positive selection, whereas $\mathrm{dN} / \mathrm{dS}$ values significantly lower than 1 suggest negative selection. In our experimental set up, only mutations that reach a minimum size are detected by deepSNV. Therefore, a significant value of $d N / d S>1$ indicates that a clone that acquires a nonsynonymous mutation in that particular gene will have a higher probability to reach a detectable size as compared to a synonymous mutation in the same gene. Hence, genes with $\mathrm{dN} / \mathrm{dS}>1$ are considered drivers of clonal expansion.

\section{Whole exome sequencing of surviving esophageal tumors.}

Sample preparation and imaging. Mice were treated with DEN in drinking water 3 times a week for 8 weeks as described above. 9-18 months after DEN removal mice were culled and the esophagus harvested. Tissues were incubated for $2-3 \mathrm{~h}$ in $5 \mathrm{mM}$ EDTA at $37^{\circ} \mathrm{C}$ before removing the submucosa from the epithelium as described above. The EEs were immunostained against KRT6 to detect the tumors and imaged on a fluorescent scope equipped with the appropriate filters. The projected area of the tumors was measured using Volocity 3D Image Analysis Software.

Tumor isolation and sequencing. Tumors were manually cut under a fluorescent microdissecting scope (Leica Microsystems) using ultra fine forceps and micro-scalpels. Individual tumors were collected in low binding DNA tubes and digested in $3 \mu$ RLT buffer (Qiagen Cat\# 1048449) for 30min at room temperature. Digested samples were diluted 1:10 in water, separated in triplicates, transferred to 96-well plates and incubated $15 \mathrm{~min}$ at room temperature with Agencourt AMPure XP magnetic beads (Beckman Coulter Cat\# A63881) at a 1:1 ratio. Beads with bound DNA were separated with a magnet and washed 3 times with $70 \%$ ethanol. DNA was re-suspended in $10 \mu$ l elution buffer and transferred to a new plate. Whole genome DNA was amplified using $1 \mu$ polymerase enzyme from the illustra GenomiPhi V2 DNA Amplification Kit (GE Healthcare Cat\# 25-6600-32) and $9 \mu$ l of sample with the following conditions: $95{ }^{\circ} \mathrm{C}$ for $3 \mathrm{~min}, 4{ }^{\circ} \mathrm{C}$ for $5 \mathrm{~min}, 30{ }^{\circ} \mathrm{C}$ for 1.5 hours and $65{ }^{\circ} \mathrm{C}$ for $10 \mathrm{~min}$. DNA was then purified by mixing with beads at a 1:0.6 DNA/beads ratio followed by 3 washes with 70\% ethanol and eluted with $30 \mu$ l of elution buffer (Qiagen Cat\# 19086). 
Whole-exome sequencing was performed using the Mouse_Exome_Targets baitset from the Wellcome Sanger Institute pipeline. Captured material was sequenced on Illumina HiSeq 2500 sequencers using paired-end $75 \mathrm{bp}$ reads.

Mutation calling and sequencing analysis. Substitutions were called using the CaVEMan (Cancer Variants through Expectation Maximization) variant caller (version v1.11.2, http://cancerit.github.io/CaVEMan) ${ }^{27}$. Insertions and deletions were called using cgpPindel (version v2.2.4, http://cancerit.github.io/cgpPindel) ${ }^{28}$. Mutations were annotated using VAGrENT (v3.3.3, https://github.com/cancerit/VAGrENT) ${ }^{28}$. Mutations occurring outside of the exome were removed by retaining only mutations annotated with the effects 'missense', 'nonsense', 'ess_splice', 'frameshift' or 'silent'. The mutational spectra and signature of single clones was analyzed as described above.

Copy number analysis of whole exome sequenced tumors. The whole exome sequencing results were used to analyze the copy number alterations of surviving tumors. We used a modified version of QDNAseq (https://github.com/ccagc/QDNAseq/) to call somatic copy number alterations ${ }^{29}$. QDNAseq was adapted to use only so-called "off-target" reads from exome sequencing, which yield an even, shallow coverage across the genome ${ }^{30}$. To obtain off-target reads, we first counted all reads per $1 \mathrm{~Kb}$ bins along the genome, then removed any bin that overlaps with the start/end coordinates of a protein-coding gene (Ensembl version GRCm38.p6) and mapped the remaining onto bins of $1 \mathrm{Mb}$. Read counting was performed separately for each tumor sample and its matched control, after which the two coverage tracks were transformed into a log ratio track (commonly referred to as "logr"). The transformation occurs similarly to that of the Battenberg copy number caller ${ }^{31}$ : first the tumor bin-counts were divided by the control bin-counts to obtain the coverage ratio; the coverage ratio was then divided by the mean coverage ratio and finally the log 2 was taken to obtain logr. The regular QDNAseq pipeline was then applied to adjust the logr for mappability and GC-content, and the genome segmented into regions of constant signal. Each segment was then subjected to a significance test to obtain copy number alterations. A t-test was applied between the logr of the segment and a logr of 0 (the expected logr if the segment is not altered), with the standard deviation of both distributions set to that of the observed data. A segment was not tested if its logr fell within [-0.05, 0.05] to conservatively account for remaining noise. The t-test had different power between segments, as these differed in size and therefore in the number of bins from which logr data was drawn. To account for this difference, we sampled up to 500 bins without replacement from the segment to perform the test. A one-sided t-test was then applied for gains and losses separately and p-values were adjusted for multiple testing using Bonferroni correction.

As every tumor was sequenced three times we applied a pipeline that used these triplicates to obtain confident copy number calls for each tumor. First, the method described above 
was applied to each sample and matched control, and we only kept alterations if they were called in all three samples that represent a tumor. This yielded 5 calls across 5 tumors. A tumor, however, had only a single matched control. This single control was used to normalize the data for all three triplicates of a tumor and could introduce false positive calls. We reasoned that a true alteration would remain when a sample is run against another control. We therefore randomly matched samples with a different control that was matched for sex, requiring each sample in a triplicate to be matched with a different control. The copy number pipeline was applied to each sample part of the 5 tumors that were found to contain an alteration in the first step, and again kept calls obtained in all three triplicates. This reduced the number of calls to 2 , spanning 2 tumors.

Whole genome sequencing of 1 year post-DEN esophageal neoplasms.

Sample preparation and sequencing. Wild type mice were treated with DEN and the esophagus collected 1 year later. EE were stained with Dapi and KRT6 to label the tumors and imaged by confocal microscopy. Neoplasms were manually cut out under a fluorescent dissecting microscope. Samples were digested and DNA isolated as above (see WES methods section) and subjected to whole genome sequencing (median coverage 3.13x), using 150-base-pair clipped reads, on a HiSeq 4000 platform (Illumina).

Copy number analysis. We applied a modified version of QDNAseq (https://github.com/ccagc/QDNAseq/) that takes into account the control that is available for each sample for an additional normalization step, to obtain calls where the coverage log ratio is significantly different from a normal diploid state. Inspection of the results revealed a number of calls in a distinct region within chromosome 12 and on chromosome $\mathrm{X}$ in samples where the control was sample MD5921b. A run of control samples against each other also revealed frequent calls on chromosome 12 , spanning a region $5 \mathrm{Mb}$ (bp $18,200,001$ to $23,300,000$ ), which was subsequently blacklisted. We further used the largest call $(1.15 \mathrm{Mb})$ in this run of control samples to establish a threshold on the minimum genomic region to be covered, and kept calls only if they exceed it ( 3 calls filtered). Inspection of control sample MD5921b revealed unusually low coverage, which suggests the gains called in samples where this control was used during analysis are likely false positives. We therefore removed calls on chromosome $X$ in these samples (14 calls filtered). After filtering, the final set of alterations contains a gain that covers the whole of chromosome 19 in sample MD5924e and a focal loss on chromosome 14 in sample MD5928e (Extended Data Fig. 6a).

Detection of apoptosis by activated caspase-3 staining. Wild-type mice received DEN in drinking water for eight weeks as described above, and culled 10 days after the treatment 
stopped. Esophagus were collected, fixed, and immuno-stained to label active caspase-3 and KRT6. Tissues were imaged by confocal microscopy using a $x 40$ objective and the presence of caspase- 3 positive cells within the tumors or in the surrounding normal epithelium was analyzed with Image $J$.

EdU lineage tracing assay. EdU (5-ethynyl-2?-deoxyuridine) incorporates into dividing cells and therefore quantification of EdU positive cells can be used as a direct measure of cell proliferation in the tissue. Mice received DEN in drinking water for 8 weeks as described above. 10 days after finishing DEN treatment mice were administered $10 \mu \mathrm{g}$ of EdU (i.p.) and the esophagus collected $1 \mathrm{~h}$ later. Tissues were peeled, fixed and the incorporated EdU detected using a Click-iT EdU imaging kit (Life technologies Cat\# C10086) according to the manufacturer's instructions. Tissues were imaged by confocal microscopy using a x40 objective and the presence of EdU positive cells in the tumors and their adjacent normal epithelium analyzed using IMARIS software.

In vivo transgenic label-retaining cell assay. Rosa $26^{M 2 r t T A} /$ TetO-HGFP mice were used to measure the rate of cell division in DEN generated tumors as compared to their adjacent normal epithelium. These animals are double-transgenic for a reverse tetracyclinecontrolled transactivator (rtTA-M2) targeted to the Rosa 26 locus and a HIST1H2BJ/EGFP fusion protein (Histone-Green Fluorescent Protein, HGFP) expressed from a tetracycline promoter element ${ }^{32}$. Administration of doxycycline (Doxy, Sigma Aldrich Cat\# D9891) induces the transient expression of HGFP, resulting in nuclear fluorescent labelling throughout the entire epithelium. When Doxy is withdrawn, HGFP is no longer expressed and is diluted lineally by half after every cell division cycle (Fig. 3b). As a result, the decline in fluorescence intensity can be used to calculate the cell division rate. Rosa26 M2rtTA $/ T e t O-$ HGFP mice received DEN for eight weeks as described above. During the last 4 weeks of DEN treatment, Doxy $(2 \mathrm{mg} / \mathrm{ml})$ was also supplied in the drinking water. After DEN-Doxy treatment, mice were culled and esophagus collected at times $\mathrm{t}=0$ (immediately after the treatments stopped), $\mathrm{t}=10$ days or $\mathrm{t}=30$ days. Tissues were peeled, fixed and stained (nuclei and KRT6) as detailed above, and imaged on a confocal microscope using a 40x objective. The intensity of HGFP in the tumors and the adjacent normal epithelia was analyzed using ImageJ.

Leukocyte density in tumors and normal epithelium. Wild-type mice were treated with DEN for 2 months and the esophagus collected 10- or 30-days after treatment. The esophageal epitheliums were processed and immuno-stained with anti-CD45 and anti-KRT6 antibodies to visualize leukocytes and tumors, respectively, and imaged on a confocal microscope. Typical settings for acquisition of multiple $z$ stacks were $2.5 \mu \mathrm{m}$ z-step size, zoom x1, optimal pinhole, line average 4, scan speed 400? $\mathrm{Hz}$ and a resolution of 1,024 $\times$ 1,024 pixels using a $10 \mathrm{X}$ HC PL Apo CS Dry objective with a 0.4NA. Visualization and image 
analysis were performed using IMARIS (bitplane) and ImageJ software. Images, with the tumors placed at the center of a $300 \times 300$ microns field of view, were used to quantify the number of leukocytes within and outside the tumors. This number was normalized by the area of the tumor or the surrounding normal tissue (calculated by subtracting the area of the tumors to the total area).

Permutation analysis of leukocyte location within tumors and normal tissue. To investigate whether the number of immune cells was enriched or excluded within the tumors, or instead followed a random distribution, we use a permutation analysis based on the experimental measurements of leukocyte density in tumors and normal epithelium obtained as detailed above. For this, tumors were assigned a circular shape, with an area matching that measured experimentally. Immune cells (CD45 ${ }^{+}$cells) were also assumed to be circular with a diameter of 8 microns. For each field of view, the location of the immune cells was left intact while the location of the tumor was randomly shuffled (ensuring the entirety of the tumor remained within the boundaries of the field of view), and the number of immune cells in contact with the tumor was counted (Extended Data Fig. 7h). This was repeated 1000 times to produce the expected distribution assuming no association between tumor and immune cell locations.

Induction of DN-Maml1 clones. Ahcre ${ }^{E R T} R 26^{f I D N M-G F P / w t}$ mice were used for induction of the dominant negative mutant of Mam/1 (DN-Maml1) ${ }^{9}$. This mutant inhibits Notch intracellular domain induced transcription, therefore disrupting the Notch signaling pathway. It is also fused to GFP, which allows for clonal labelling of the mutant. Expression of DN-Maml1 can be genetically induced following treatment with ß-napthoflavone (BNF, MP Biomedicals Cat\# 156738) and tamoxifen (TAM, Sigma Aldrich Cat\# N3633). Specifically, transcription of the Cre mutant estrogen receptor fusion protein (CreERT) is induced following intraperitoneal (i.p) BNF injection. A subsequent i.p injection of TAM is necessary in order for the CreERT protein to gain access to the nucleus and excise the loxP flanked "STOP" cassette resulting in the expression of DN-Maml1 tagged to GFP. As the switch occurs at the gene level, the descendants of the originally labelled cell (clones) will also constitutively express DN-Maml1, and can be visualized by fluorescent microscopy.

Mice received DEN for 8 weeks as described above. 10 days after DEN withdrawal, mice were given a single injection of BNF (80?mg? $\mathrm{kg}$ ) and TAM (1? $\mathrm{mg}$ ), or left un-induced. Esophagus were collected 30 days post-DEN treatment (20 days after induction of DNMaml1 clones). Whole tissues were processed, stained with anti-GFP and KRT6 antibodies and imaged on a confocal microscope as described above. The percentage of esophageal epithelium occupied by DN-Maml1 mutant clones and the number of tumors within and outside DN-Maml1 ${ }^{+}$areas were measured using Volocity 3D Image Analysis Software (Perkin Elmer) or IMARIS software. 
Percentage of tumors eliminated by DN-Maml1 clones. The percentage of tumors removed by expanding DN-Maml1 clones was calculated as follow: \% of tumors eliminated $=(1-$ $T /$ (DNneg)) 100. Where $T$ is the total number of tumors per $\mathrm{mm}^{2}$ of esophageal epithelium and DNneg is the number of tumors per $\mathrm{mm}^{2}$ of DN-Maml1 negative epithelium within the same tissue.

In vivo treatment with the $\boldsymbol{\gamma}$-secretase inhibitor DBZ. Wild-type mice received DEN in drinking water for 8 weeks as described above. 10 days after DEN withdrawal, mice were injected i.p. with the $\gamma$-secretase inhibitor DBZ (S2711; Selleckchem) at $30 \mu \mathrm{mol} / \mathrm{Kg}$ of bodyweight, using a stock solution of $2.8 \mathrm{mg} / \mathrm{ml}$ in $0.5 \%$ hydroxypropyl methylcellulose (Methocel 65HG, 64670-100G-F, Sigma) and 0.1\% Tween-80 in water. Control animals were injected with vehicle solution. Mice received DBZ or vehicle every three days, a total of 5 injections, and were culled 2 weeks after initiating the treatment ( 24 days post-DEN). Esophagus were collected and epitheliums from control and DBZ-treated mice processed and stained with KRT6 antibodies. Whole-mounted esophageal epitheliums were imaged by confocal microscopy and the number of tumors per $\mathrm{mm}^{2}$ of epithelium measured using IMARIS software.

Fitting of model to data. See Supplementary information. The parameter sets which best fit the data were found using Approximate Bayesian Computation based on sequential Monte Carlo sampling (ABC-SMC) ${ }^{33}$ implemented in the python package pyABC ${ }^{34}$. This method randomly generates parameter combinations, then uses summary statistics to compare the model's output using those parameters to the observed data, and rejects parameter combinations for which the model's output is too dissimilar to the data. Multiple generations are run with increasingly stringent thresholds for acceptance, with each new generation of parameters based on the accepted parameter combinations from the previous generation. In this manner, the method identifies the regions of parameter space that produce model outputs most similar to the observed data. We ran 25 generations with a population of 10000 (the number of accepted parameter combinations in each generation). For the summary statistic, we used the total squared distance of the mean model prediction from the mean observed tumor density. For the tumor drift model with no interaction with mutant clones in surrounding normal tissue, the fit was to the tumor density following DEN-treatment. For the model including clone interaction, the fit was to the tumor density following DEN-treatment and the tumor density in the DBZ experiment. Uniform distributions using the bounds in Supplementary information Table A (see Supplementary information) were used as the initial prior distributions of the parameters.

Statistical analysis. Data are expressed as mean values \pm s.e.m. unless otherwise indicated. No statistical method was used to predetermine sample size. The experiments were not 
randomized. The investigators were not blinded to allocation during experiments and outcome assessment.

Data availability. Individual data sets are available in Supplementary Tables 1-8. The accession number for the DNA targeted sequencing of 10 days and 1 year tumors is ENA: ERP022921. Accession numbers for WES and WGS of tumors are ENA: ERP015469 and ENA: ERP122780, respectively.

Code availability. Code is available at https://github.com/michaelhall28/Colom lesions.

Acknowledgments: We thank Esther Choolun, Tom Metcalf and staff at the MRC ARES and Sanger RSF animal facilities for technical support. This work was supported by grants from the Wellcome Trust to the Wellcome Sanger Institute (098051 and 296194) and Cancer Research UK Programme Grants to P.H.J. (C609/A17257 and C609/A27326). B.A.H. and M.W.J.H. are supported by the Medical Research Council (Grant-in-Aid to the MRC Cancer unit grant number MC_UU_12022/9 and NIRG to B.A.H. grant number MR/S000216/1). A.H. benefited from the award of an EMBO long term fellowship. M.W.J.H. acknowledges support from the Harrison Watson Fund at Clare College, Cambridge. B.A.H. acknowledges support from the Royal Society (grant no. UF130039). S.D. benefited from the award of an ESPOD fellowship, 2018-21, from the Wellcome Sanger Institute and the European Bioinformatics Institute EMBL-EBI. K.T.M. benefited from the support of the Chan Zuckerberg Initiative. We are grateful to the Cambridge Biorepository for Translational Medicine for access to human tissue.

Author contributions: B.C., M.P.A. and A.H. designed experiments. B.C., A.H., M.P.A., G.P. and D.F.A performed experiments and analyzed data. B.C., M.W.J.H., S.C.D., C.K., R.K.S. and S.H.O. analyzed sequencing data. M.W.J.H. developed the mathematical modelling. K.T.M., K.S.P., and J.C.F. collected human samples. B.C., M.W.J.H., and P.H.J. wrote the paper. B.A.H., M.G. and P.H.J. supervised the research.

Competing interests: Authors declare no competing interests. 

available under aCC-BY 4.0 International license.

Supplementary information is available for this paper: see below

Correspondence and requests for materials should be addressed to P.J.H. 


\section{Supplementary information}

This document describes the whole exome sequencing (section 1) and analysis of chromosomal number alteration (section 2) of surviving tumors, as well as the experimental work to explore potential mechanism of elimination of early tumors such as tumor cell apoptosis and loss of proliferation (section 3), and the immune system (section 4). The theory and mathematical modeling of tumor dynamics is then set out (section 5).

\section{Section 1. Whole exome sequencing (WES) of surviving tumors.}

The $\mathrm{dN} / \mathrm{dS}$ analysis of targeted sequenced samples indicated that 5 out of the 192 genes analyzed, Atp2a2, Notch1, Notch2, Chuk and Adam10 are significantly $(q<0.05)$ positively selected in tumors persisting 1 year after DEN treatment (Fig. 2c). To explore whether other genes not included in our targeted sequencing bait set were selected in these samples, we performed whole exome sequencing (WES) of persisting tumors.

Wild type mice were treated with DEN and esophagus collected 9-18 months later (Extended Data Fig. 5a). EE were stained with Dapi and antibodies against KRT6 to label neoplasms and imaged by confocal microscopy. 49 tumors from 16 tissues were identified, manually cut out under a fluorescent dissecting microscope and digested. Genomic DNA was isolated from each sample and split into three pools, each of which underwent independent whole genome amplification (WGA) and WES to an average cumulative coverage of 1130x (Extended Data Fig. 5a-b). To exclude artefactual SNVs generated during WGA, only mutations shared by all three amplified triplicates were included in the subsequent analysis.

After applying these criteria a total of 32,736 mutations were identified, including silent, missense, nonsense and splice mutations and indels (Supplementary Table 6). The median number of SNVs/exome per isolated tumor was 669, ranging from 56 to 1256, with an average variance allele fraction (VAF) of $0.233 \pm 0.124$ (mean \pm SD) (Extended Data Fig. 5cd). The spectrum and functional impact of mutations was consistent with the results obtained with the targeted sequencing approach (Extended Data Fig. 5e-f). Finally, dN/dS analysis indicated that Atp2a2 and Notch1 were positively selected in these samples (Extended Data Fig. $5 \mathrm{~g}$ and Supplementary Table 7).

\section{Section 2. Analysis of DNA copy number alterations (CNAs) in 1 year tumors.}

CNAs are rare in normal human esophageal epithelium but common in esophageal cancers $2,5,35$. Thus, we explored whether the presence of CNAs would be a feature of the surviving tumors at 1 year post-DEN treatment. 
We initially performed whole genome sequencing (WGS) of 64 tumors from 9 esophagus, with a median coverage of 3.13x. Control samples obtained from the same animals were used for an additional normalization step. After applying some custom filters (see Methods) only two tumors showed evidence of chromosomal alterations, where the coverage log ratio was different from a normal diploid state. These include a gain of the whole chromosome 19 and a focal loss on chromosome 14 (Extended Data Fig. 7a).

We next interrogated our WES dataset to look for CNAs, to ensure the above WGS was not underpowered due to the polyclonality of these samples ${ }^{15}$ (section 1). Somatic CNAs were called from the WES tumors as reported before ${ }^{11}$. In line with the results obtained with WGS, the analysis of WES samples showed negligible presence of CNAs in the surviving tumors. Only 2 of the 49 tumors analyzed exhibited evidence of alterations, particularly in Chromosomes 7 and 16 (Extended Data Fig. 7b-c).

\section{Section 3. Early neoplasms are not eliminated by increased apoptosis or decreased proliferation of tumor cells.}

Here we set out how we investigated apoptosis and the rate of proliferation in murine normal and neoplastic EE.

To determine whether the elimination of early tumors was related to increased apoptosis of tumor cells, wild-type mice were treated with DEN for two months and the esophagus collected 10 days after the last treatment. Tissues were then immuno-stained against KRT6, to label tumors, and activated Caspase-3 as a maker of apoptosis, and imaged by confocal microscopy (Fig. 3a) In line with our previous observations we found negligible activated Caspase-3 positive cells in the DEN-treated normal epithelium ( 0.04\% of cells) ${ }^{11}$. Importantly, no Caspase-3 positive cells were detected within the tumors, suggesting that early neoplasms are not lost through apoptosis.

Another potential mechanism for the elimination of early tumors could be the loss of proliferating tumor cells. To assess this, we first injected EdU (5-ethynyl-2? -deoxyuridine) into mice, 1 hour before tissue collection (Extended Data Fig. 7a). EdU incorporates into replicating DNA and labels cells in S phase of the cell cycle. We found that cells within the tumors incorporate EdU to a similar extent than their surrounding normal epithelium (Extended Data Fig. 7b). We next measured the rate of cell division in transgenic assay using Rosa $26^{M 2 r t T A} /$ TetO-HGFP mice. In this strain, expression of a stable Histone-Green Fluorescent Protein (HGFP) is regulated by a Doxycycline-responsive transgenic promoter. When Doxycycline is withdrawn, levels of the stable HGFP protein fall at a rate dependent on the rate of cell division (Fig. 3b) ${ }^{13,15,32}$. Rosa $26^{\text {M2rtTA }} /$ TetO-HGFP mice received DEN for 4 weeks followed by DEN + Doxycycline for another 4 weeks. Animals were culled and tissues 
collected at times 0, 10 days and 30 days after DEN + Doxycycline withdrawal (Fig. 3c). Analysis of the intensity of HGFP in tumors gradually decreased with time (Fig. 3d and Extended Data Fig. 7c). Importantly, the rate of HGFP loss at each time point was not significantly different between the tumors and the adjacent normal epithelium, indicating that cells within the tumors divided at a similar rate than the surrounding normal epithelium.

From these results we conclude that micro-tumors were not lost due to apoptosis or abnormal proliferation of tumor cells.

\section{Section 4. Early neoplasms are not eliminated by the immune system.}

We next explored whether micro-tumors were recognized and eliminated by the immune system. If this was the case, then tumors would not be removed as efficiently in mice with a defective immune system. We tested this using NOD.Cg-Prkdc ${ }^{\text {scid }} \| 12 \mathrm{rg}^{\text {tmI } 1 w_{j l} /} / \mathrm{SzJ}^{22}$ mice, a strain that lacks typical anti-tumor immune responses due to a complete absence of $B$ and $T$ lymphocytes and NK cells, as well as defective dendritic cells and macrophages. NOD.Cg-

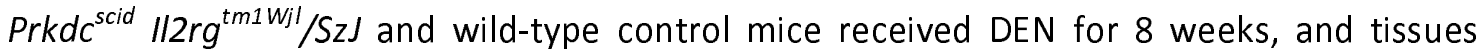
were collected 10 and 30 days post-DEN withdrawal, stained and analyzed by confocal microscopy (Fig. 3e). There was no significant difference between strains in the number of tumors lost (Fig. 3f). This indicates that tumors are removed even if the immune response is severely compromised. Of note, the lack of a significant difference in the number of tumors at 10 days also suggests that the immune system does not have a critical role in the formation of tumors in this model.

To further explore the relationship between immune cells and early tumors we analyzed the spatial localization of leukocytes ( $\mathrm{CD} 45^{+}$cells) in EEs from DEN-treated wild-type mice collected 10 days and 30 days post-DEN withdrawal (Extended Data Fig. 7d). The results show a positive correlation between the size of the tumors and the number of immune cells within them (Extended Data Fig. 7e). Furthermore, our analysis indicates that the number of immune cells normalized by tumor area positively correlates with that in the adjacent normal tissue (Extended Data Fig. 7f). Consequently, no significant differences were found in the average number of immune cells present within the tumors and at the surrounding normal EE at either the 10 or 30 day time points (Extended Data Fig. 7g). These results argue that the presence of immune cells within early neoplasms follows the same pattern than the adjacent normal tissue, suggesting that immune cells are not able to detect the tumors at this early stage.

Finally, we used a different approach to investigate whether immune cells were enriched, excluded or behaved neutrally with respect to the early tumors. For this purpose, we performed a permutation analysis taking advantage of the known spatial localization of the 
immune cells in the images from the above experiments. For each field of view, the coordinates of the immune cells were fixed, the tumor randomly shuffled within the image and the number of immune cells in contact with the "phantom" tumors quantified (Extended Data Fig. 7h). The observed number of immune cells in contact with the tumors differed slightly from the calculated numbers due to the assumed circularity of the tumors and immune cells (Extended Data Fig. 7i and Methods). Despite this, the number of immune cells in contact with tumors was not significantly different from a random distribution (Extended Data Fig. 7j). We conclude that immune cells are not enriched or selected but behave neutrally with respect to early micro-tumors.

All together, these results argue that immune cells are not responsible for the early elimination of tumors.

\section{Section 5 . Theory and mathematical modeling}

\section{Modelling aims}

Here we describe a general model of tumor loss that allows us to make testable predictions without requiring details of cell dynamics in tumors or mutant clones in the epithelium. We are interested in particular in whether the tumors are lost due to tumor-intrinsic mechanisms, or if the tumors are removed by mutant clones in the adjacent normal epithelium. As we discuss the factors affecting tumor loss, the principles of the model are described in mathematical terms so that we can later construct a numerical version of the model. This numerical version of the model is intended as a demonstration of the model principles, rather than a parameter- or model-fitting exercise.

In section 5.1, we briefly summarize previous results regarding growth and competition of mutant clones in normal EE. In section 5.2, we describe a previously proposed stochastic model of tumor dynamics and apply it to the tumor density following DEN-treatment. In section 5.3, we show that tumor loss is affected by the induction of highly fit mutant clones in the surrounding tissue. In section 5.4, we show how reducing the competitive imbalance between tumors and highly fit mutant clones in the normal tissue increases the number of surviving tumors. In section 5.5, we discuss how certain tumor genotypes may have higher survival chances due to a resistance to displacement by mutant clones in the normal tissue. In section 5.6, we substitute simple mathematical equations into the model to numerically illustrate the principles described in the previous sections. Section $\mathbf{5 . 7}$ is a brief summary of our conclusions

\subsection{Clonal competition in esophageal epithelium.}


Before examining the behavior of tumors in the tissue, we first summarize previously published results relating to competition of mutant clones in phenotypically normal murine $\mathrm{EE}^{11}$.

The EE is maintained by proliferating cells in the basal layer (Extended Data Fig. 1a). These cells divide stochastically (randomly) (Extended Data Fig. 1b), meaning that, if we track the offspring of basal cells, some cell lineages will grow into multicellular clones of the original cell, and other lineages will be lost as all basal cells differentiate ${ }^{13}$. A mutation in a basal cell may convey a growth advantage, biasing cell fate towards producing more dividing daughter cells and promoting the growth of a mutant clone ${ }^{9-12,36}$ (Extended Data Fig. 1c).

When the tissue contains multiple mutant clones, the clones compete for the limited space in the tissue ${ }^{11}$ (Extended Data Fig. 1d). Fitter clones are able to displace less fit clones, but once a clone is surrounded by clones of similar competitive fitness it returns towards more neutral growth ${ }^{11}$ (Extended Data Fig. 1d). The expansion and survival of a mutant clone therefore depends not only on its own fitness, but the fitness of its neighbors ${ }^{11}$.

Following DEN treatment, the density of tumors in the EE falls rapidly (Fig. 1d). Tumors are not lost due to chromosomal copy number alterations (Extended Data Fig. 7 and section 2 above), apoptosis (Fig. 3a and section $\mathbf{3}$ above), loss of proliferation (Fig. 3d, Extended Data Fig. 7b-c and section 3 above) or interaction with the immune system (Fig. 3f, Extended Data Fig. 7d-j and section 4 above). The tumors grow amongst a dense patchwork of highly fit clones competing for their place in the tissue. In the following sections we explore whether the tumors survival is affected by the mutant clones around them.

\subsection{Stochastic model of tumor dynamics.}

In a previous study we found that the growth of high grade dysplasias (HGDs) in the esophagus of DEN and Sorafenib treated mice was consistent with a stochastic model of cell dynamics ${ }^{15}$. In this model, proliferating cells divide to form a pair of proliferating daughter cells, a pair of non-dividing daughter cells, or one cell of each type (Extended Data Fig. 1bc). In the normal tissue, the probabilities of each symmetric division type are balanced, so that the total number of proliferating cells remains approximately constant (Extended Data Fig. 1b). In the HGDs, there was a bias towards producing more dividing than differentiated cells, causing the average size of tumors to increase over time (Extended Data Fig. 1c) ${ }^{15}$.

Due to the stochastic nature of the process, some tumors expand in size, while in others all basal cells differentiate and the tumor is shed and lost from the tissue ${ }^{15,37}$. This variation in outcome due to random chance is known as drift. If we assume that the fully differentiated tumors are lost from the tissue, then the survival probability of a tumor is given by the equation $^{38}$ : 


$$
p_{\text {surv }}(t)=1-\alpha^{n} \#(1)
$$

where $n$ is the initial number of proliferating cells in each tumor (assumed here to be the same for all tumors), and

$$
\alpha=\frac{\left(\frac{1}{2}-\Delta\right)\left(e^{2 d \Delta t}-1\right)}{\left(\frac{1}{2}+\Delta\right) e^{2 d \Delta t}-\left(\frac{1}{2}-\Delta\right)}
$$

Where $d$ is the symmetric division rate of tumor cells, $\Delta$ is the probability imbalance between symmetric division and symmetric differentiation (Extended Data Fig. 1c), and $t$ is time. Fitting equation 1 to the tumor density following DEN-treatment results in a steep initial drop in tumor numbers followed by a slower downward trend, consistent with the experiment (Supplementary information Figure A). The median accepted parameters found from fitting the model to the data (see Methods) were (95\% credible interval lower bound, upper bound) $d=0.33 /$ day $(0.27,0.36), \Delta=0.003(0.001,0.005), n=1.2(1.0,1.5)$, and initial tumor density (immediately following DEN-treatment) $=4.8 / \mathrm{mm}^{2}(4.1,5.6)$, though these parameters should not be interpreted as estimates of the true biological values (see

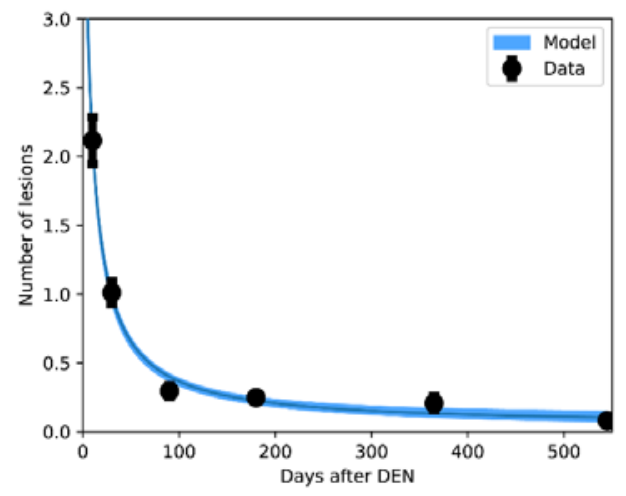

Supplementary information Figure A. Tumor drift model (with no interaction with mutant clones in surrounding normal tissue) fit to tumor density (\# tumors per $\mathrm{mm}^{2}$ of $\mathrm{EE}$ ) following DEN-treatment (see Methods). The mean value and range between the minimum and maximum values of the models run with the accepted parameters are shown.

sections below).

However, we will see in the sections below that this model is not capable of explaining the results seen in the full range of experiments, and therefore must be rejected (or at least adjusted) to account for the clones in the surrounding tissue.

\subsection{Elimination of tumors by highly competitive mutant clones in the surrounding normal epithelium.}


Although the stochastic model of tumor drift defined above is sufficient to describe the pattern of tumor loss following DEN-treatment, it does not rule out alternative causes of tumor loss. The normal EE surrounding the tumors contains a patchwork of competing mutant clones (see section 5.1). Therefore, we speculated that, like clones in the surrounding normal tissue, tumors may be displaced by highly fit mutant clones.

We considered a general model in which a highly competitive mutation, $M$, is induced in the tissue following DEN treatment. We assume that clones of the mutant $M$ are able to remove tumors that they encounter (Extended Data Figs. 8a and 9c). Let $p_{M}(t)$ be the survival probability of a tumor assuming that the mutant $M$ is the only cause of tumor loss. Let $p_{\text {other }}$ be the survival probability of a tumor based on all other sources of tumor loss (such as drift - see section 5.2, but may include tumors removed by DEN-created clones - see section $\mathbf{5 . 4}$ below). For simplicity, we assume that tumor loss due to the mutant $M$ is independent of all other causes of tumor loss. The combined survival probability of a tumor is then given by

$$
p_{\text {surv }}(t)=p_{\text {other }}(t) \times p_{M}(t) \#(2)
$$

Let $M(t)$ be the proportion of tissue covered by the mutant at time $t$. We make the following additional assumptions for the sake of simplicity:

1. The proportion of tissue covered by $M$ increases monotonically.

2. Tumors are spread randomly across the tissue.

3. Tumors are removed instantly with probability 1 when the mutant $M$ colonizes the location of the tumor.

This means

$$
p_{M}(t)=1-M(t) \#(3)
$$

and the combined probability of tumor survival is then given by

$$
p_{\text {surv }}(t)=p_{\text {other }}(t) \times(1-M(t)) \#(4)
$$

To make it easier to compare mice in which the initial density of tumors may vary, we looked at the proportion of lesions eliminated $(P L E)$ by $M$, using the tumor density in the 
non-M-mutant regions of the tissue to estimate the tumor density in the full tissue in the absence of $M$.

$$
P L E=1-\frac{\text { total }}{\text { Mneg }} \#(5)
$$

where total is the density of tumors over the full tissue, and Mneg is the density of lesions in the M-negative region.

The expected PLE for two models - where the $M$ mutant removes lesions it encounters $\left(P L E_{M}\right)$ and where tumor loss is independent of the mutant in the surrounding tissue $\left(P L E_{\neg M}\right)$, are

$$
\begin{gathered}
P L E_{M}=1-\frac{p_{\text {surv }}(t)}{p_{\text {other }}(t)}=M(t) \#(6) \\
P L E_{\neg M}=1-\frac{p_{\text {other }}(t)}{p_{\text {other }}(t)}=0 \#(7)
\end{gathered}
$$

In other words, if $M$ clones are able to remove tumors in a similar manner to which highly fit clones are able to displace weaker clones in the normal tissue, then we will see a reduction in tumor numbers proportional to the spread of the $M$ mutant. By definition, models in which tumor survival is independent of surrounding tissue $\left(P L E_{\neg M}\right)$, such as the stochastic tumor drift model described in section 5.2, predict that tumor numbers will be unaffected by the spread of the $M$ clones (Supplementary information Figure B). 


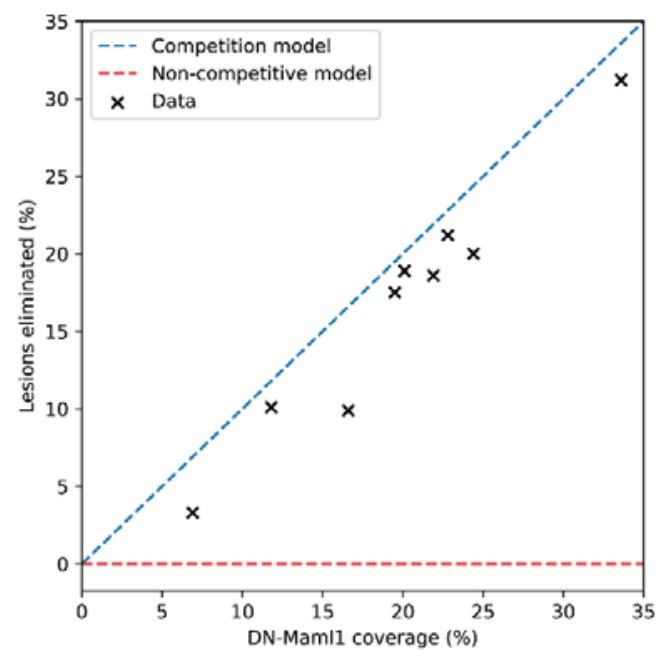

Supplementary information Figure B. Tumor loss when highly fit mutant clones (such as DN-Maml1 clones) are induced in EE following DEN-treatment. Predictions of a model in which the induced mutant clones immediately remove lesions they encounter (blue) and a model in which tumor loss is independent of clones in the surrounding tissue (red).

To test these predictions, we used an inducible DN-Maml1 mutation that prevents Notch signaling and forms rapidly expanding clones when induced in murine $\mathrm{EE}^{11}$. The data shows a strong correlation between DN-Maml1 clone spread and tumor loss, indicating that DNMaml1 clones are indeed removing tumors from the tissue (Fig. 4c-d, Supplementary information Figure B).

In the experiment, there remained a small number of tumors in close contact with DNMaml1 mutant regions (Fig. 4e). This may indicate that there is a lag time between contact with DN-Maml1 clones and tumor removal and that the surviving tumors seen in the DNMaml1 areas are in the process in being removed (Fig. 4 e and Extended Data Fig. 9a-c). It may also be the case that a small proportion of tumors are able to survive despite the competition with DN-Maml1 clones (see section 5.5 below).

\subsection{Reducing competitive imbalance.}

Now that we have shown that the induction of a highly fit mutant following DEN treatment can eliminate tumors, we asked whether mutant clones already present in the DEN-treated tissue are able to remove tumors too. Fit clones present in the normal epithelium might be able to out-compete the tumors, eliminating them from the tissue. By removing the competitive advantage of those clones, we can examine the impact they are having on tumor survival. 
We assume there is a type of highly fit mutant clone, $N$, in the DEN-treated tissue that is able to remove tumors. As in the section above (Equation 2), we assume that the removal of clones by mutant clones $N$ is independent of other causes of tumor loss. Tumor survival probability is given by

$$
p_{\text {surv }}^{\text {ctl }}(t)=p_{\text {other }}(t) \times p_{N}^{c t l}(t) \#(8)
$$

where, similar to above, $p_{\text {other }}(t)$ is the survival probability of a tumor based on all sources of tumor loss other than elimination by $N$ clones, and $p_{N}^{c t l}(t)$ is the survival probability of a tumor assuming that the mutant $N$ is the only cause of tumor loss. We assume that, without intervention, $N$ clones spread progressively throughout the tissue and outcompete tumors they encounter, so $p_{N}^{c t l}\left(t_{2}\right)<p_{N}^{c t l}\left(t_{1}\right)$ for $t_{2}>t_{1}$.

If we can raise the fitness of the surrounding tissue and tumors to a similar level as $N$ clones, this would both prevent the spread of the $N$ clones across the tissue (reducing the number of tumors directly competing with $N$ clones) and reduce the elimination of tumors that are already adjacent to $N$ clones, as they will now be competing neutrally (Extended Data Fig. 10a). Assuming that we have an intervention that completely levels the fitness of $N$ clones with the rest of the tissue and tumors, the loss of tumors due to $N$ clones will cease during that period, i.e. if the intervention starts at time $t_{1}$ and lasts until $t_{2}, p_{N}^{\text {int }}\left(t_{2}\right)=p_{N}^{\text {int }}\left(t_{1}\right)=$ $p_{N}^{c t l}\left(t_{1}\right)>p_{N}^{c t l}\left(t_{2}\right)$ and

$$
p_{\text {surv }}^{\text {int }}\left(t_{2}\right)=p_{\text {other }}\left(t_{2}\right) \times p_{N}^{\text {int }}\left(t_{2}\right)>p_{\text {other }}\left(t_{2}\right) \times p_{N}^{\text {ctl }}\left(t_{2}\right)=p_{\text {surv }}^{\text {ctl }}\left(t_{2}\right)
$$

where, for the experiment in which the intervention is applied, $p_{\text {surv }}^{\text {int }}(t)$ is the overall tumor survival probability and $p_{N}^{i n t}(t)$ is the tumor survival probability related to the mutant $N$. Therefore, if we can remove or reduce the competitive imbalance between a mutant that can remove tumors and the rest of the tissue (including the tumors), then we should see an increase in surviving tumors compared to control experiments (Extended Data Fig. 10b).

Notch1 mutant clones dominate competition in the normal EE ${ }^{11}$ and therefore is a good candidate mutation to test this prediction. The Notch inhibitor Dibenzazepine (DBZ) prevents Notch signaling and would affect all cells in the tissue, effectively raising the fitness of all Notch wild type clones and tumors to the level of the Notch mutant clones ${ }^{9}$. The competitive advantage of Notch mutants is thus removed during the DBZ intervention. Furthermore, by raising all cells to a high background level of fitness, this may also reduce the relative fitness advantage conveyed by mutations which work independently of the Notch pathway ${ }^{39}$. 
We indeed saw the predicted increase in surviving tumors when DBZ is administered between 10 days $\left(t_{1}\right)$ and 24 days $\left(t_{2}\right)$ after DEN treatment (Extended Data Fig. 10c-d and Supplementary information Figure $\mathrm{C}$ ). This suggests that competition from mutant clones in the surrounding tissue is removing a substantial proportion of the tumors lost in the first

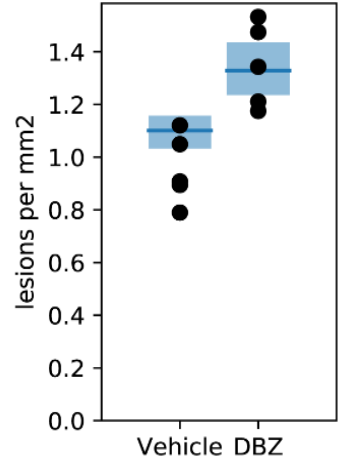

Supplementary information Figure C. Numerical example of the model (see section 5.6) showing increased tumor survival by administration of DBZ as compared to vehicle control. Model fit to lesion density data using Approximate Bayesian Computation (see section $\mathbf{5 . 6}$ and Methods). The mean value and range between the minimum and maximum values of the model run with the accepted parameters are shown.

few weeks following DEN treatment.

\subsection{Selection pressure on tumors from competition with surrounding mutant clones.}

There are genetic differences between the tumors sequenced 10 days and 1 year after DEN treatment, as shown by the $\mathrm{dN} / \mathrm{dS}$ ratios (Fig. $\mathbf{2 c}$ ) and the proportion of tumors mutant for each selected gene (Extended Data Fig. 4g). This genetic change over time could be consistent with ongoing selection of mutant subclones within tumors ${ }^{40}$. However, the elimination of early neoplasms by mutant clones in the surrounding tissue could also act as a selective pressure on tumors.

As described in section $\mathbf{5 . 4}$ above, the competitive fitness of a tumor compared to the surrounding clones will affect the survival prospects of the tumor (Fig. 4a). If certain tumor genotypes have a competitive fitness comparable to or higher than the fittest mutant clones in the surrounding tissue, they would be more likely to survive. For example, Notch1 is the dominant mutant gene in normal tissue, occupying almost the entire tissue 12 months after DEN treatment ${ }^{11}$. We might expect that, similar to the Notch1 mutant clones in the normal tissue, tumors which are also Notch1 mutant would be able resist displacement by the mutant clones in the surrounding tissue. Consistent with this, we saw an increase in the proportion of Notch1 mutant tumors from the 10-day to the 1-year time point (Extended Data Fig. $4 \mathrm{~g}$ ). We also see a large increase in the proportion of Atp2a2 mutant tumors (Extended Data Fig. 4g), suggesting that these too may be able to resist displacement by clones in the surrounding tissue. 
To explore this hypothesis, we expanded the model to include two tumor phenotypes: sensitive and resistant. Sensitive tumors can be removed by clones in the surrounding tissue, and resistant clones cannot. The probability of tumor survival is then given by

$$
p_{\text {surv }}(t)=S(0) \times p_{\text {other }}(t) \times p_{N}(t)+R(0) \times p_{\text {other }}(t) \#(9)
$$

where $S(0)$ and $R(0)$ are the proportions of tumors at day 0 following DEN treatment which are sensitive and resistant respectively.

The proportion of surviving tumors which are resistant to displacement by mutant clones, $R(t)$, is given by

$$
R(t)=\frac{R(0)}{R(0)+S(0) p_{N}(t)} \#(10)
$$

Assuming that the number of sensitive and resistant tumors are non-zero, and that, as we assumed earlier, $p_{N}(t)$ is a decreasing function of time, then the proportion of surviving tumors which are resistant will increase over time.

The increasing proportion of the surviving tumors which are Notch1 and/or Atp2a2 mutant is consistent with the hypothesis that these mutations may increase the tumor's ability to resist displacement by clones in the surrounding tissue. The presence of a subset of tumors which are able to resist displacement by clones also could explain why a small fraction of tumors are able to survive over 1 year after DEN treatment (Fig. 1d) when the surrounding normal tissue is almost entirely populated by highly fit mutant clones ${ }^{11}$.

\subsection{Numerical model example.}

So far, we have mostly defined properties of the tumor survival probabilities rather than given specific equations for their values. This has allowed us to make testable predictions without requiring the details of the clonal or tumor dynamics to be defined.

Here we substitute feasible functions into Equation 9 to construct a numerical expression of the model. The functions are intended to be simple and introduce only a small number of model parameters, and the purpose here is to illustrate the concepts described in the previous sections rather than accurately and verifiably model the data. 
Firstly, we need to define $p_{\text {other }}$, the non-clone related probability of tumor survival. As we have shown that apoptosis and abnormal proliferation of tumor cells, and the immune system are not contributing to tumor loss (Fig. 3, Extended Data Fig. $\mathbf{7}$ and sections $\mathbf{3}$ and $\mathbf{4}$ above), we assume that $p_{\text {other }}$ is simply the survival probability based on drift (Equation 1 ). Secondly, we need to define $p_{N}(t)$, the probability of tumor survival based on highly fit mutant clones in the surrounding tissue. Following the assumptions in sections $\mathbf{5 . 4}$ and 5.5, we assume that there is a single mutant population $N$ capable of removing tumors and that $N$ mutant clones remove tumors as soon as they occupy the location of the tumor in the tissue. Our starting time occurs after the end of DEN treatment, and so much of the tissue may already be occupied by the $N$ clones. Therefore we modify Equation 3 to account for the sensitive tumors existing in the remaining non- $N$ proportion of the tissue.

$$
p_{N}(t)=\left(\frac{1-N(t)}{1-N(0)}\right) \#(11)
$$

This still leaves us having to define $N(t)$, the growth pattern of the mutant clones. Growth of mutant clones in EE have previously been modelled using branching processes ${ }^{12}$, but these don't consider competition between clones and limitations of the tissue size ${ }^{11,41}$. Cellular automaton simulations have also been used to model clones in $\mathrm{EE}^{11}$, but this does not allow for easy integration with the mathematical formulation. Instead, we used the logistic equation, which captures the key features of clonal spread: fast growth at early time points when mutant cells are mostly competing with surrounding wild type cells, slower growth at late time points when the tissue is already largely mutant, and an upper bound on total mutant spread ${ }^{11}$. Therefore,

$$
N(t)=\frac{1}{1+\left(\frac{1-x_{0}}{x_{0}}\right) e^{-k t}} \#(12)
$$

where $x_{0}$ is the initial proportion of tissue covered by $N$ mutant clones and $k$ is the clone growth rate. To represent the experiment in which the DBZ Notch inhibitor is applied between 10 and 24 days after DEN, we can define

$$
N_{D B Z}(t)=\left\{\begin{array}{cc}
N(t) & t<10 \\
N(10) & 10 \leq t \leq 24
\end{array} \#(13)\right.
$$

The full model can then be constructed by substituting these expressions into equation 9 . 
We have 7 independent parameters: $d, \Delta, n, x_{0}, k, S(0)$, and the initial tumor density (Supplementary information Table A). We used Approximate Bayesian Computation ( $A B C$ ) to find the parameter combinations for which the model most closely matched the mean tumor density from 10 days to 18 months after DEN treatment and the mean tumor density in CTL and DBZ experiments at 24 days after DEN treatment (see methods). The parameters were constrained as listed in Supplementary information Table A. The results are shown in Supplementary information Figures $\mathbf{D}$ and $\mathbf{E}$. The median acceptable parameters found were (95\% credible interval lower bound, upper bound) $d=0.29 /$ day $(0.17,0.35), \Delta=0.020$ (0.007, 0.047), $n=1.4(1.0,2.5), x_{0}=0.80(0.42,0.99), k=0.032(0.019,0.040), s(0)=0.64(0.44$, 0.82 ) and initial tumor density $=4.2 / \mathrm{mm}^{2}(3.4,5.4)$, although, given the simplifications and approximations used in this numerical example of the model, they should not be interpreted as estimates of their biological counterparts.
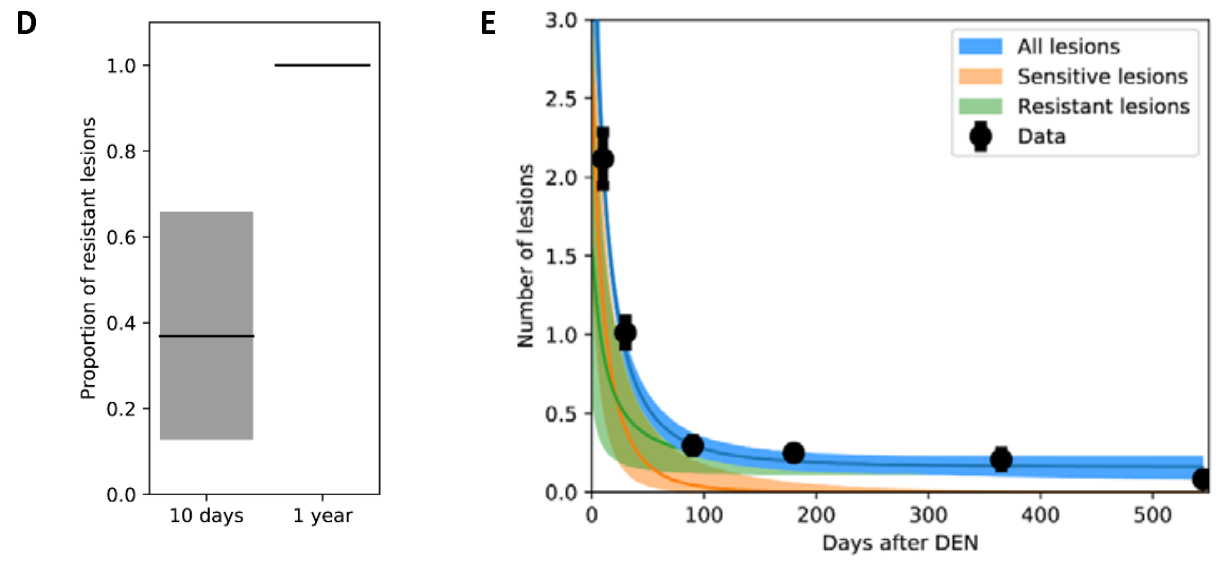

Supplementary information Figures $D$ and $E$. Numerical example of the model showing increased proportion of tumors resistant to displacement by mutant clones (D) and the decrease in lesion density (\# tumors per $\mathrm{mm}^{2}$ of EE) following DEN-treatment (E). Model fit to tumor density data using Approximate Bayesian Computation (supplementary information and Methods). The mean value and range between the minimum and maximum outputs of the model run with the accepted parameters are shown.

The numerical example of the modelling principles demonstrates how the elimination of (a subset of) tumors by mutant clones in the surrounding tissue leads to the experimental observations of decreasing tumor numbers following DEN treatment, higher tumor survival when competitive imbalance is removed (DBZ experiment), and a selection pressure on tumor genotype.

\subsection{Summary}

Together, the experimental data and modelling indicates that tumors in the DEN-treated EE are eliminated by mutant clones in the surrounding normal tissue. The data also suggests 
that the tumor genotype influences the chance of a tumor surviving, possibly by allowing the tumors to better compete with mutant clones in the surrounding tissue.

\section{Supplementary text Table A}

\begin{tabular}{|c|c|c|c|c|}
\hline Parameter & Definition & $\begin{array}{l}\text { Lower } \\
\text { bound }\end{array}$ & $\begin{array}{l}\text { Upper } \\
\text { bound }\end{array}$ & Notes \\
\hline$d$ & $\begin{array}{l}\text { Symmetric division of } \\
\text { proliferating tumor cells }\end{array}$ & 0 & 2.5/week & $\begin{array}{l}\text { Histone dilution revealed the tumors had a } \\
\text { similar division rate to the normal tissue } \\
\text { (Fig. 3d and Extended Data Fig. } 7 \text { c) } \\
\text { (approximately } 2.5 \text { times a week }{ }^{11} \text { ). This } \\
\text { was used as an upper bound on the rate of } \\
\text { symmetric divisions }\end{array}$ \\
\hline$\Delta$ & $\begin{array}{l}\text { Cell fate imbalance of } \\
\text { tumorcells }\end{array}$ & -0.5 & 0.5 & \\
\hline$n$ & $\begin{array}{l}\text { Number of proliferating } \\
\text { cells per tumor at end of } \\
\text { DEN treatment. }\end{array}$ & 1 & 100 & \\
\hline$x_{0}$ & $\begin{array}{l}\text { Proportion of tissue } \\
\text { covered by tumor-removing } \\
\text { clones at the end of the } \\
\text { DEN treatment }\end{array}$ & 0 & 1 & \\
\hline$k$ & $\begin{array}{l}\text { Growth rate of tumor- } \\
\text { removing clones }\end{array}$ & 0 & 0.04 & $\begin{array}{l}\text { Upper bound is the fit to DN-Maml1 } \\
\text { mutant clone expansion in wild type } \\
\text { background (data from }{ }^{9} \text { ). }\end{array}$ \\
\hline$S(0)$ & $\begin{array}{l}\text { Initial proportion of tumors } \\
\text { that can be removed by } \\
\text { clones in the surrounding } \\
\text { tissue. }\end{array}$ & 0 & 1 & \\
\hline $\begin{array}{l}\text { Initial } \\
\text { tumor } \\
\text { density }\end{array}$ & $\begin{array}{l}\text { tumors per } \mathrm{mm}^{2} \text { at the end } \\
\text { of DEN treatment. }\end{array}$ & 0 & 20 & $\begin{array}{l}\text { At } 10 \text { days, there are approximately } 2 \\
\text { tumors per } \mathrm{mm} 2 \text { (Fig. 1d). Allowed upper } \\
\text { bound to be order of magnitude higher } \\
\text { immediately following DEN treatment. }\end{array}$ \\
\hline
\end{tabular}



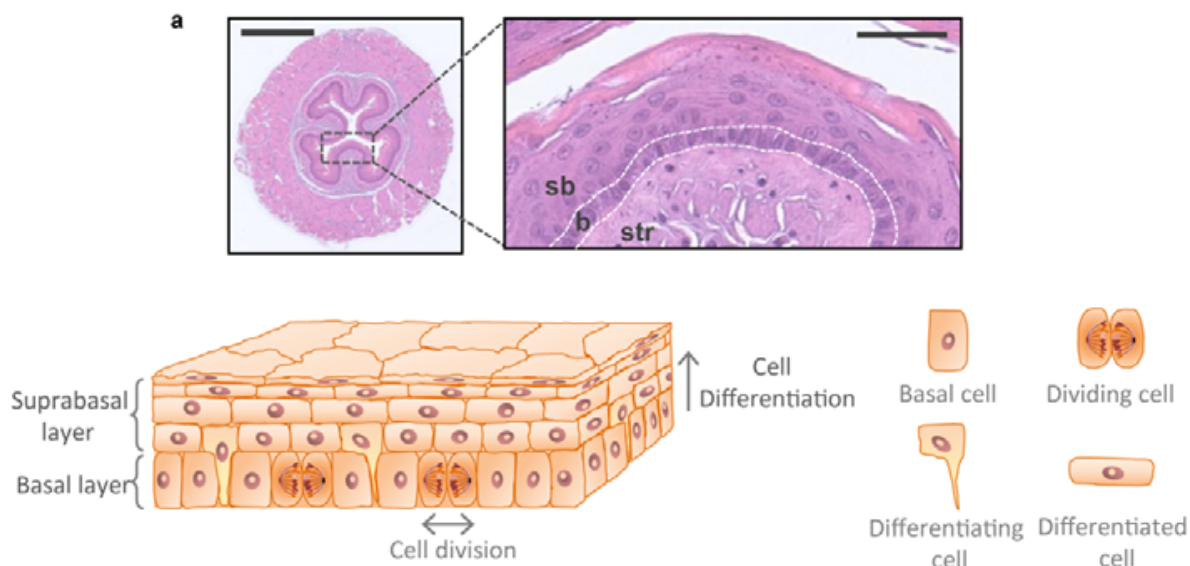

b Outcome after division
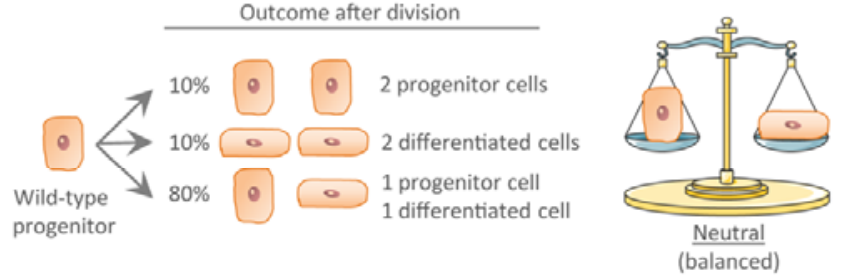

Net outcome:

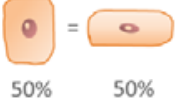

c
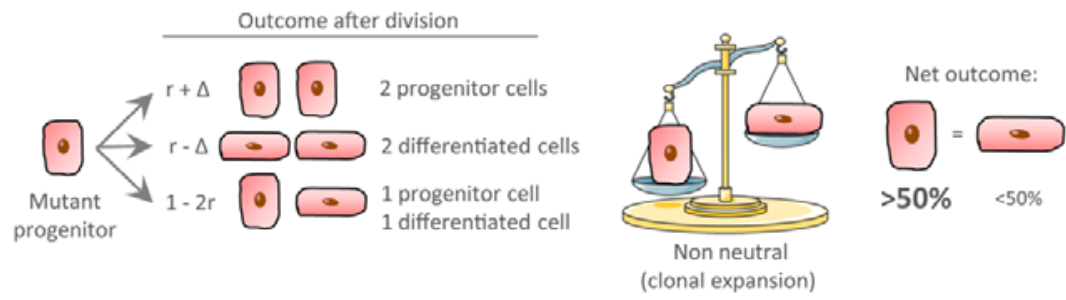

d
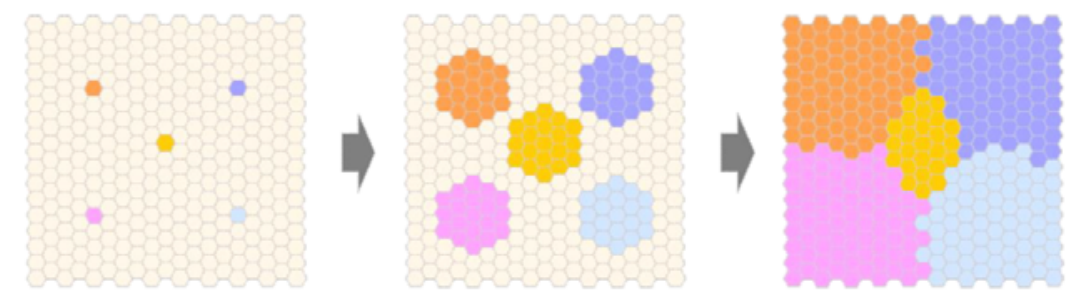

Extended Data Fig. 1. Mouse EE architecture and dynamics. (a) H\&E images and schematic of the mouse EE architecture and dynamics; $b=$ basal cell layer (delineated by white dots), sb = suprabasal layers, str $=$ stroma. Scale-bars: $500 \mu \mathrm{m}$ (top), $50 \mu \mathrm{m}$ (bottom). Dividing (progenitor) cells are confined to the basal layer. Differentiating cells exit the cell cycle, migrate out of the basal layer, through the suprabasal layers, and are finally shed. (b-c) The single progenitor model (Supplementary information). All progenitor cells in the EE basal layer are functionally equivalent and following division produce either: two progenitors that will persist in the tissue, two differentiating cells that will cease division, stratify and be lost or one cell of each type. In homeostasis (b), the likelihood of the two progenitor and two differentiating cell outcomes is equal. Mutations (c) may tip the balance towards a non-neutral behavior, resulting in clonal growth if they favor proliferation of daughter cells. (d) Expansion of mutant clones is defined by their relative competitive fitness to adjacent clones. Initially, a fit "winner" mutant progenitor (colored) shows a fate bias towards proliferation and outcompetes its less fit "loser" surrounding cells, resulting in clonal expansion. Eventually, mutant clones begin to collide with each other until surrounded by similarly competitive mutants, at 
bioRxiv preprint doi: https://doi.org/10.1101/2021.06.25.449880; this version posted June 25, 2021. The copyright holder for this preprint (which was not certified by peer review) is the author/funder, who has granted bioRxiv a license to display the preprint in perpetuity. It is made available under aCC-BY 4.0 International license.

which point their cell fate reverts towards balance and their expansion slows (Supplementary information). 
bioRxiv preprint doi: https://doi.org/10.1101/2021.06.25.449880; this version posted June 25, 2021. The copyright holder for this preprint (which was not certified by peer review) is the author/funder, who has granted bioRxiv a license to display the preprint in perpetuity. It is made available under aCC-BY 4.0 International license.

a

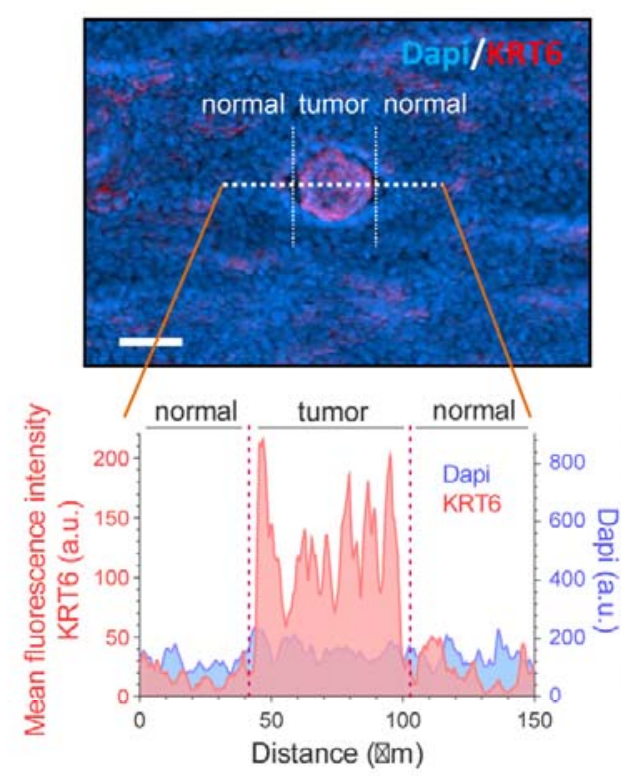

b
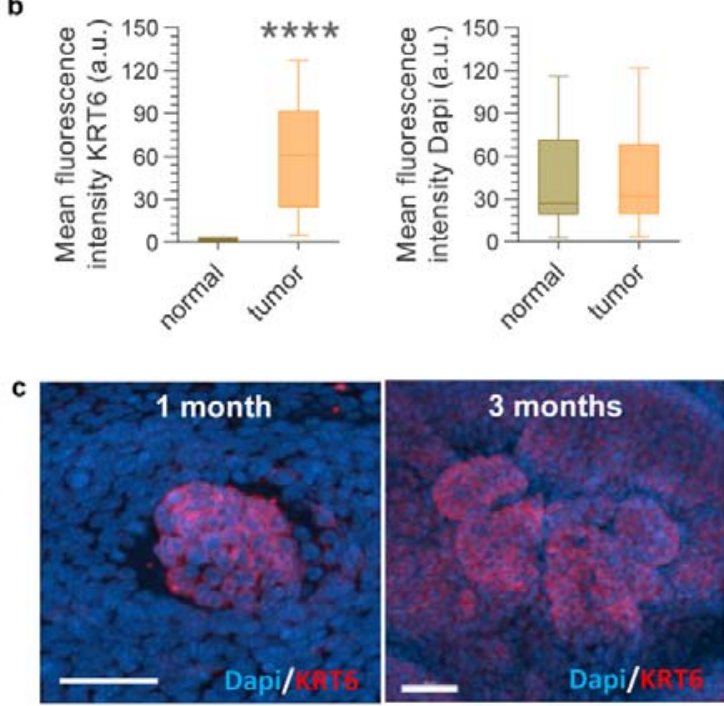

6 months

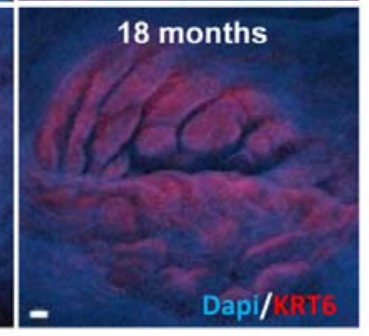

e

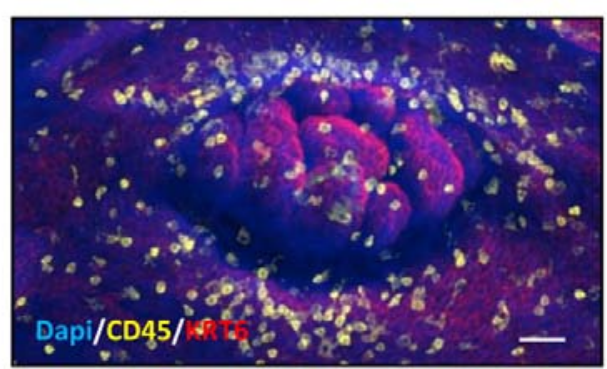

Extended Data Fig. 2. Characterization of DEN-generated tumors. (a) Image of a tumor (10-day post-DEN) stained with KRT6 and Dapi. Graph show the mean fluorescence intensity of KRT6 and Dapi measured across the white dotted line in the image above, illustrating the expression levels in the tumor and the adjacent normal epithelium. (b) Quantification of the mean fluorescence intensity of KRT6 and Dapi in tumors and their normal adjacent epithelium (as in a). ${ }^{* * * P<0.0001 ~(T w o-s i d e d ~}$ Mann-Whitney test, $n=48$ tumors from 3 mice). (c) Confocal images of mouse EE tumors collected at the indicated time points post-DEN treatment. (d) Confocal image of a 3-month post-DEN tumor stained for the endothelial cell marker CD31. Top and bottom images show top and lateral projections, respectively. (e) Confocal image of a 3-month post-DEN tumor stained for the immune cell pan-marker CD45. Scale-bars: $50 \mu \mathrm{m}$. 

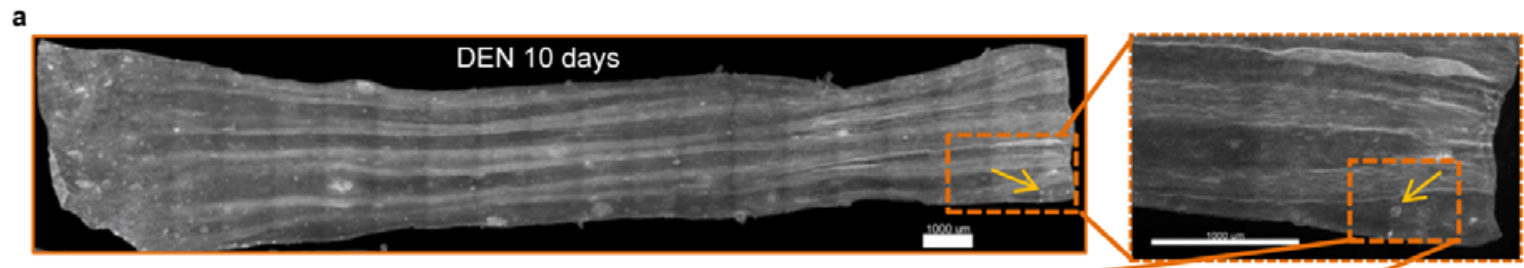

b
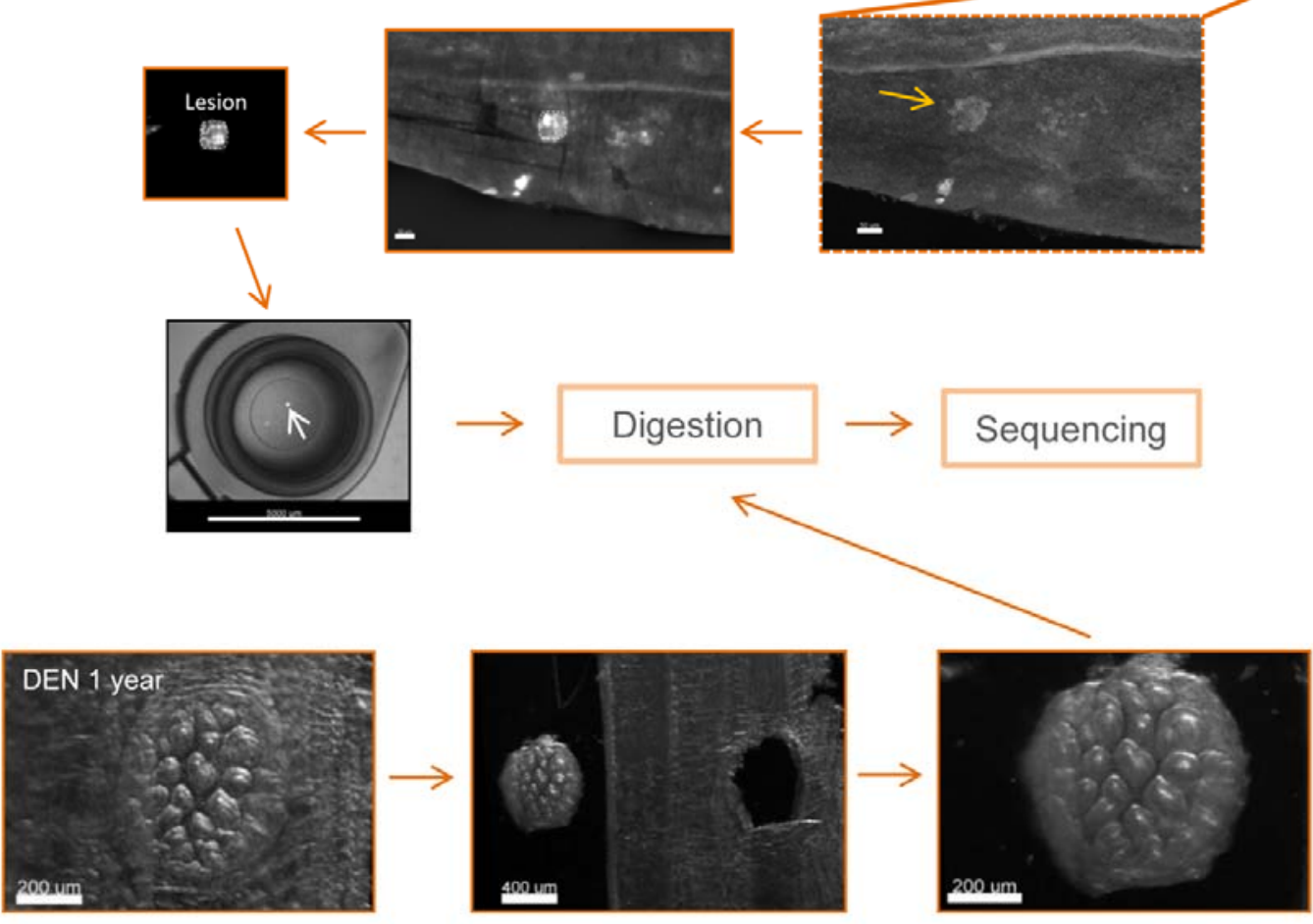

Extended Data Fig. 3. Collection of 10-day and 1-year EE tumors. (a-b) Esophagus were collected 10-days (a) or 1-year (b) post-DEN treatment. Tissues were longitudinally cut opened and the epithelium separated from the underlying muscle and stroma. Whole EEs were flattened, fixed, stained with KRT6 (grey), mounted and 3D-imaged on a confocal microscope. Tumors were identified from the processed images and manually cut under a fluorescent microscope. Samples were placed on $0.2 \mathrm{ml}$ tubes, digested and sequenced as detailed in Methods. 


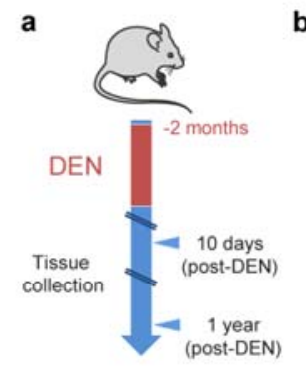

b

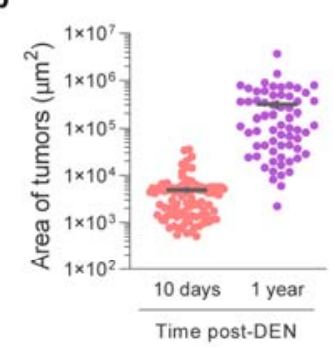

C

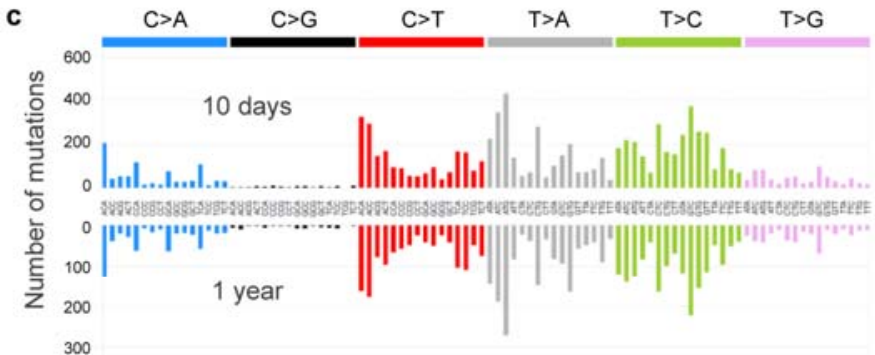

d Silent $\square$ Missense $\square$ Nonsense $\square$ Splice $\square$ Indels $\square$

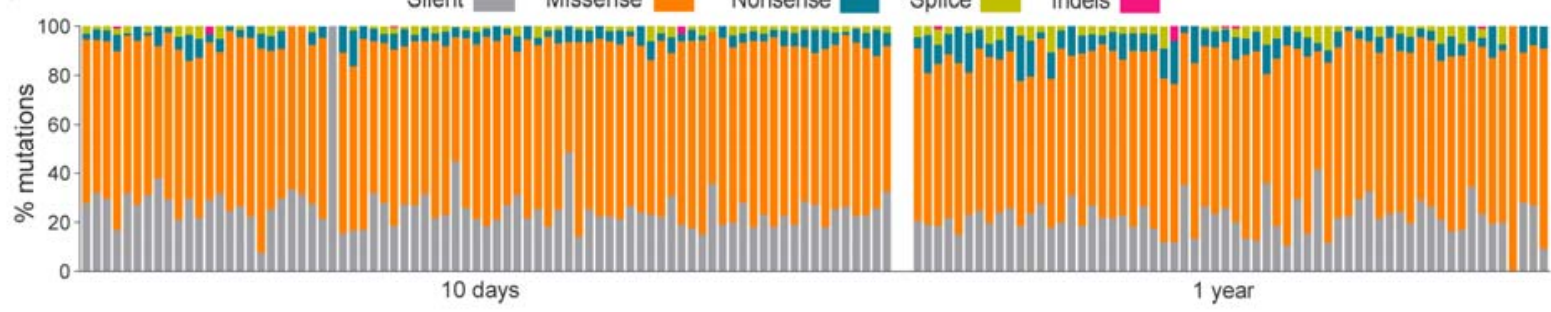

e

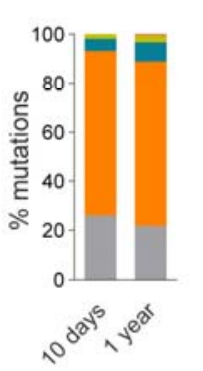

f

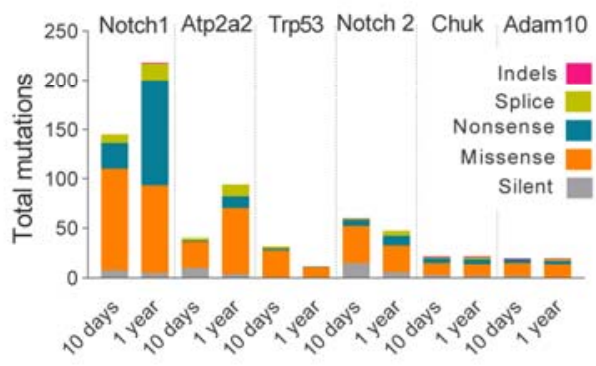

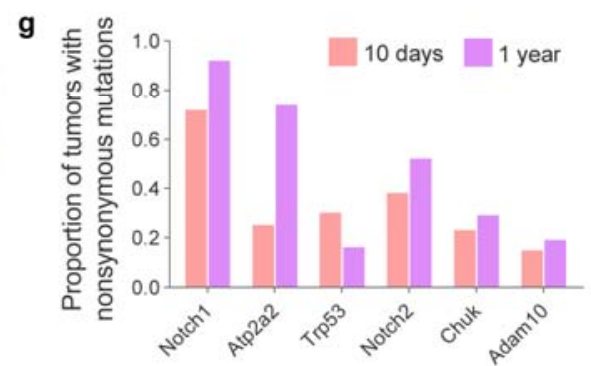

Extended Data Fig. 4. Targeted sequencing of 10 days and 1 year tumors. (a) Protocol: wild-type mice were treated for two months with DEN and the EE tumors collected 10-days or 1-year later. Samples were sequenced with a targeted approach (192 gene panel). (b) Area of 10-day and 1-year post-DEN tumors. Lines show Mean \pm s.e.m. ( $n=89$ and 64 tumors, respectively). (c) Mutational spectrum of 10-day and 1-year tumors. The bar plots illustrate the number of mutations in each of the 96 possible trinucleotides. (d-e) Percentage of silent, missense, nonsense and splice mutations and indels identified in 10-day or 1-year tumors. Graphs show the values for individual tumors (d, each column is a tumor), or the average for all tumors at each time point (e). (f) Number and type of mutations in the positively selected genes identified by $\mathrm{dN} / \mathrm{dS}$ analysis from 10-day or 1-year tumors. (g) Proportion of 10-day and 1-year tumors carrying nonsynonymous mutations in the positively selected genes. 
a

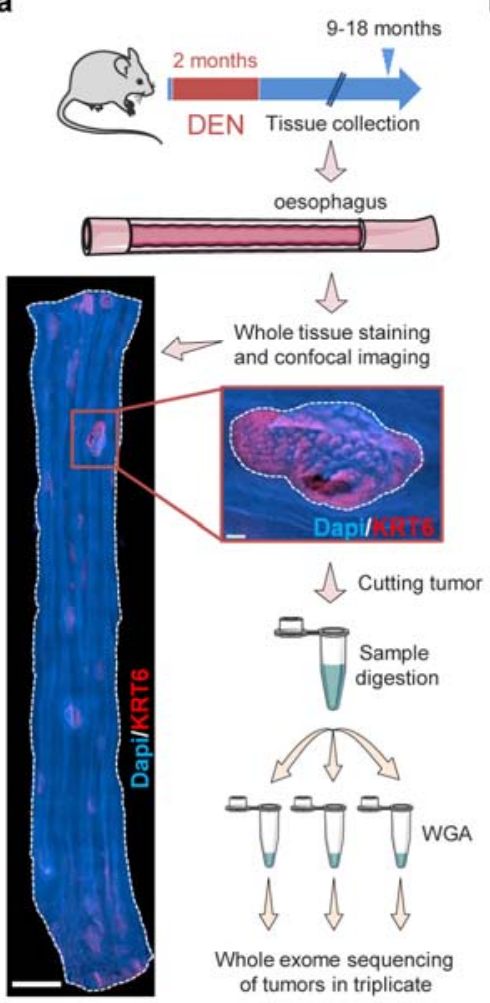

b

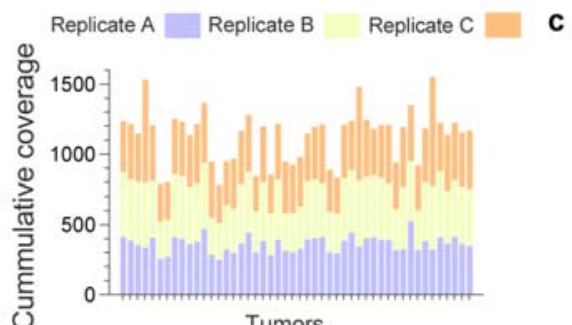

d

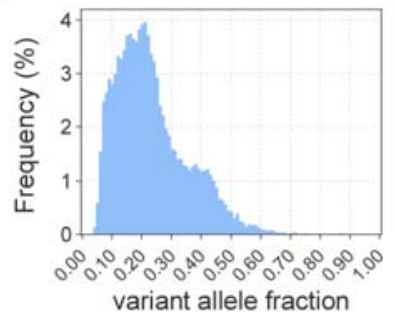

e

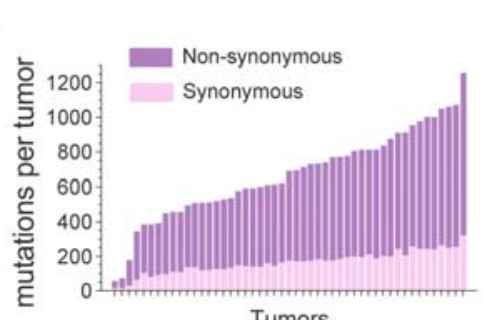

Tumors
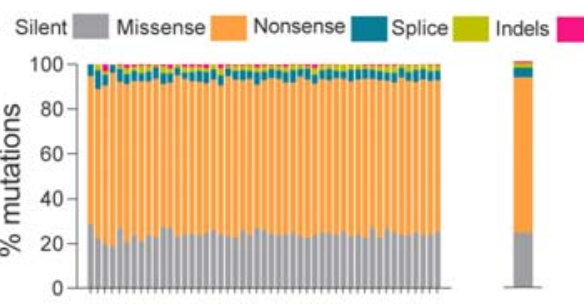

Tumors

All tumors

f

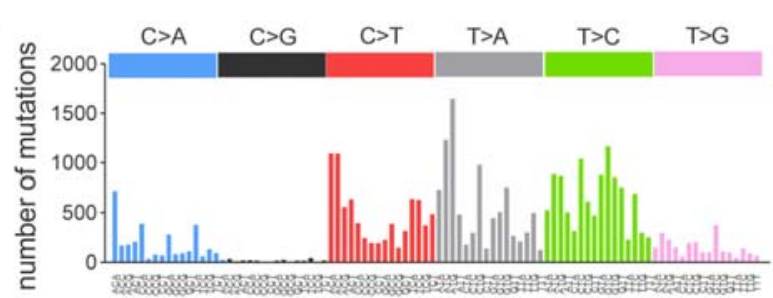

g

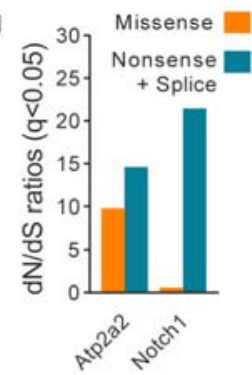

Extended Data Fig. 5. Whole exome sequencing of surviving tumors. (a) Wild-type mice received DEN for two months and the esophagus was collected 9-18 months after treatment. Tissues in (b) were stained for Dapi (blue) and KRT6 (red) and confocal imaged to identify tumors. Scale-bars = $2 \mathrm{~mm}$ (main), $150 \mu \mathrm{m}$ (inset). Individual EE tumors were manually cut under a fluorescent microscope, digested and separated in triplicates. Each triplicate was whole genome amplified (WGA) followed by whole exome sequencing. To exclude artefactual SNVs generated during WGA, only mutations shared by all three amplified triplicates were considered for further analysis. A total of 32,736 mutations, including silent, missense, nonsense and splice mutations and indels were identified from 49 tumors in 16 mice. (b) Cumulative sequencing coverage of the whole exome triplicate samples. (c) Number of synonymous (light colored) and non-synonymous (dark colored) mutations per tumor, ranked by mutation burden. (d) Distribution of the variant allele fraction (VAF) for the mutations common within triplicates in each tumor. (e) Percentage of silent, missense, nonsense and splice mutations and indels for individual tumors and for all tumors combined. (g) Mutational spectrum of the tumors. The bar plots illustrate the percentage of mutations in each of the 96 possible trinucleotides. (g) $\mathrm{dN} / \mathrm{dS}$ ratios for missense and truncating (nonsense + essential splice site) substitutions indicating genes under significant positive selection in the tumors $(q<0.05$, calculated with $\mathrm{R}$ package $\mathrm{dNdSCv}{ }^{16}$ ). 
bioRxiv preprint doi: https://doi.org/10.1101/2021.06.25.449880; this version posted June 25,2021 . The copyright holder for this preprint (which was not certified by peer review) is the author/funder, who has granted bioRxiv a license to display the preprint in perpetuity. It is made available under aCC-BY 4.0 International license.
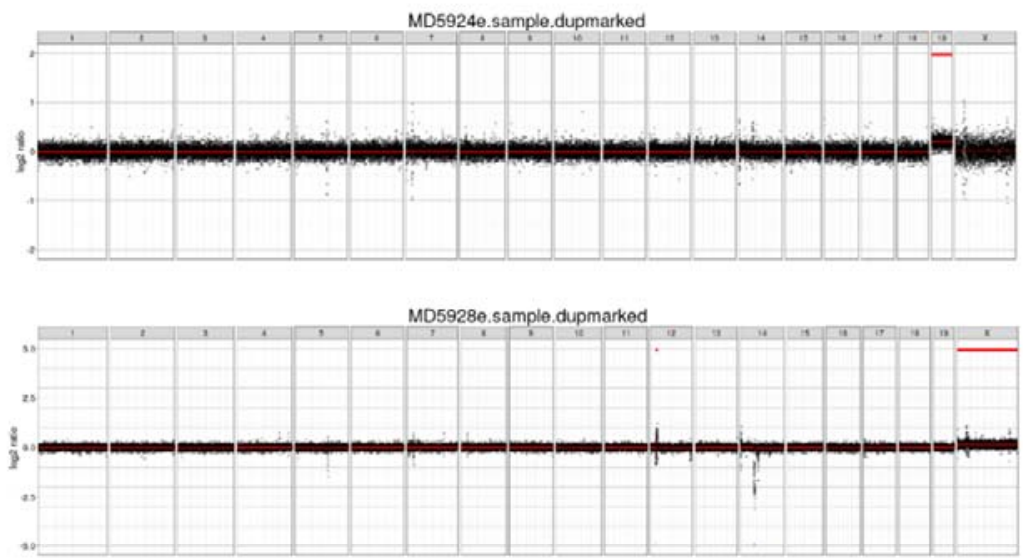

b

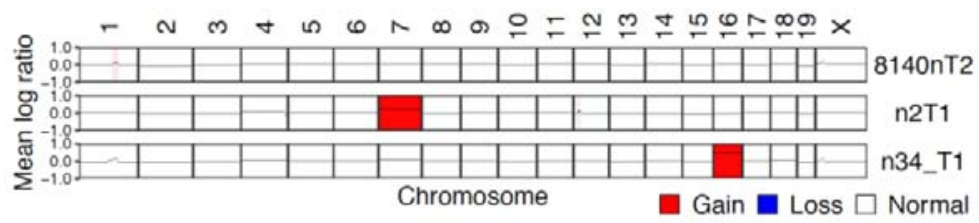

c Lesion $8140 \mathrm{nT2}$

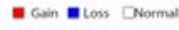
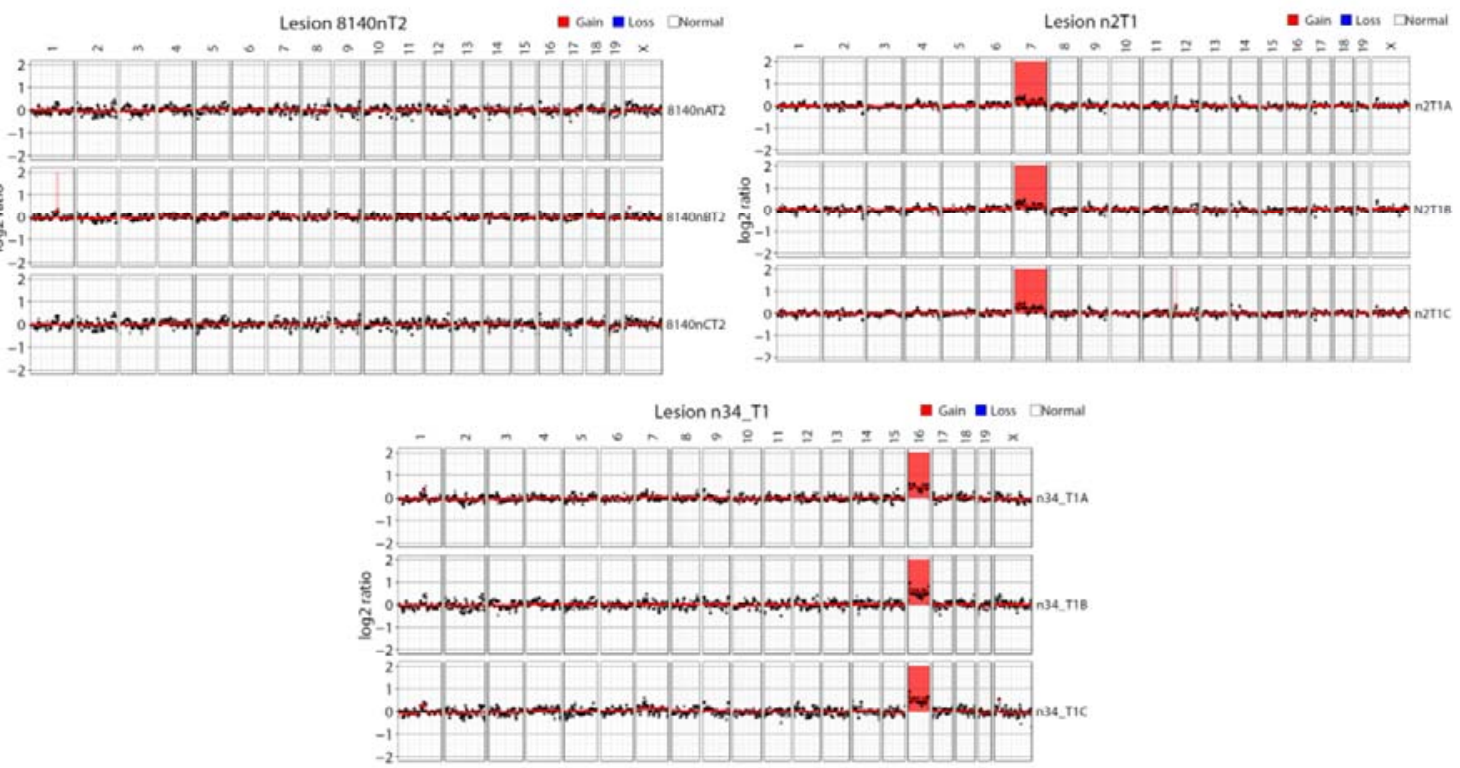

Extended Data Fig. 6. Analysis of chromosomal alterations in 1-year tumors. (a) Analysis of CNAs by whole genome sequencing of 1 year post-DEN tumors. Only 2 out of 64 tumors, MD5924e and MD5928e, exhibited CNAs. (b) Summary of chromosomal alterations found by whole exome sequencing data of 1-year post-DEN tumors in triplicate. $8140 \mathrm{nT2}$ (top) is a representative example of a tumor without chromosomal alterations. 2 out of 49 tumors ( $\mathrm{n} 2 \mathrm{~T} 1$ and $\mathrm{n} 34$ _T1) showed small alterations (indicated by color). Only alterations present in all 3 triplicates (c) were considered valid calls. 
a
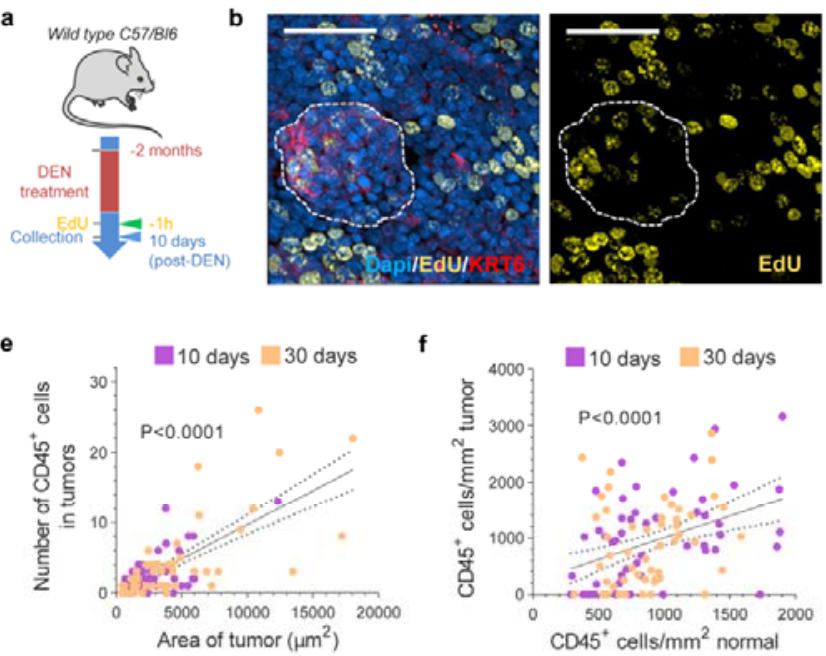

h

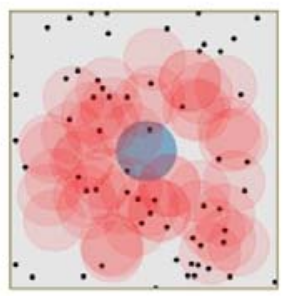

f
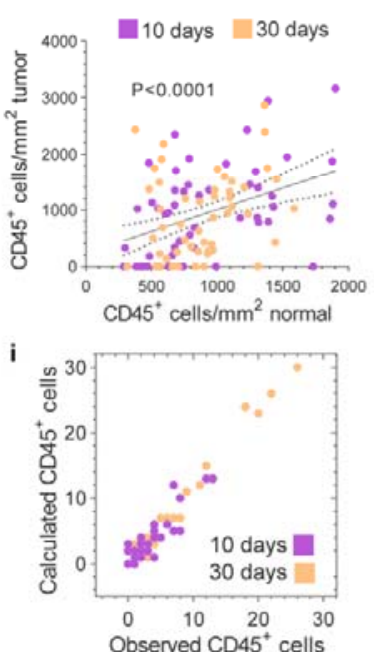

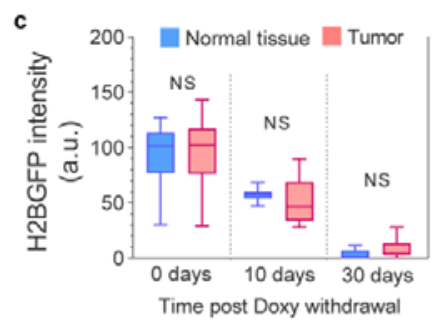

g
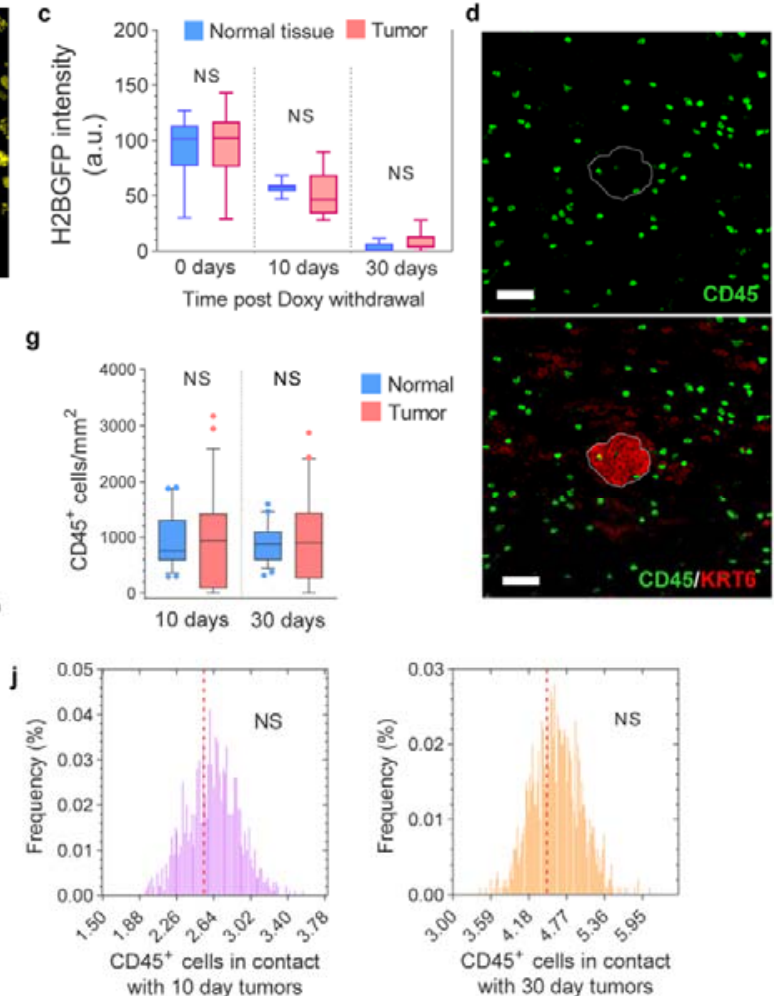

Extended Data Fig. 7. Early neoplasms are not eliminated by tumor cell abnormal proliferation or the immune system. (a) Protocol: wild-type mice were treated with DEN for two months and the tissues collected ten days later. Mice received EdU $1 \mathrm{~h}$ before tissue collection. (b) Confocal images showing EdU incorporation (1h pulse) into tumors (dotted line) and the surrounding normal epithelium ( $n=22$ tumors, 3 mice). Scale-bars: $20 \mu$ m. See Supplementary information. (c) HGFP intensity in tumors and normal EE at the indicated time points post-Doxy withdrawal. (Two-sided Mann-Whitney test, $n=10$ images per group). (d) Confocal image depicting CD45 $5^{+}$(immune) cells (green) within a 10-days post-DEN tumor (white dotted line) and its adjacent normal epithelium. (ef) Correlation between the size of the tumors and the number of $\mathrm{CD} 45^{+}$cells within them (e) and the number of $\mathrm{CD}^{+} 5^{+}$cells in the tumor and the normal epithelium (f). Lines shows the Pearson correlation: $R^{2}=0.5039(\mathrm{e})$, and $R^{2}=0.1464(\mathrm{f})$, with $95 \%$ confidence interval (dotted lines). $(\mathrm{g}$ ) Quantification of the number of $\mathrm{CD} 5^{+}$cells per $\mathrm{mm}^{2}$ of tumor and normal EE from wild-type mice 10- or 30-days post-DEN treatment (Two tailed Wilcoxon matched-pairs test, $n=53$ and 50 images, respectively). (h) Permutation analysis of leukocyte location within tumors, based on the experimental measurements obtained from (d). For each image, the location of $\mathrm{CD}_{4} 5^{+}$cells (black dots) was left intact while the location of the tumor (colored circles) was randomly shuffled (blue shows original location, red shows shuffled "phantom" tumors), and the number of immune cells in contact with the tumor was counted. This was repeated 1000 times to produce the expected distribution, assuming no association between tumor and immune cell locations. (i) Calculated (within the original location) vs. experimentally observed number of $\mathrm{CD}^{+} 5^{+}$cells in 10 - or 30-days post-DEN tumors. (j) Distribution of the average number of CD45+ cells in 10- or 30-days post-DEN tumors, obtained from the permutation analysis in (h). Red dotted line shows the experimentally observed average number of $\mathrm{CD}^{+} 5^{+}$cells within tumors at the indicated time-point. See Supplementary information. Box-plots in (c) and (g) show median (central box line) $25^{\text {th }}-75^{\text {th }}$ percentiles (box), $1^{\text {st }}-99^{\text {th }}$ percentiles (whiskers) and outliers (dots). NS= not significant. 
a

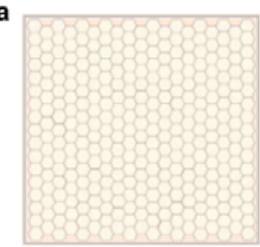

Non DN-Maml1 clones

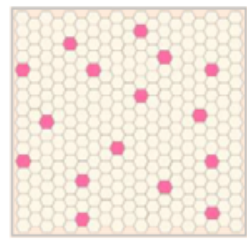

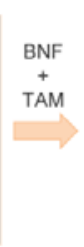

DN-Maml1 clones
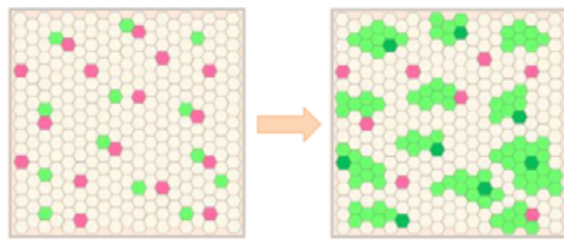

Tumors lost through DN-Maml1 clonal expansion b

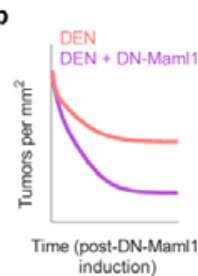

C

d
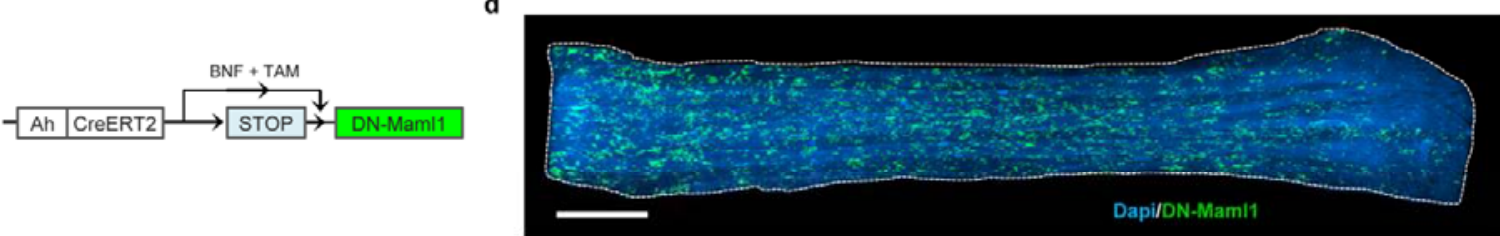

e

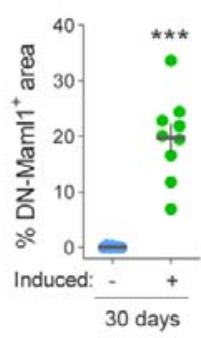

f

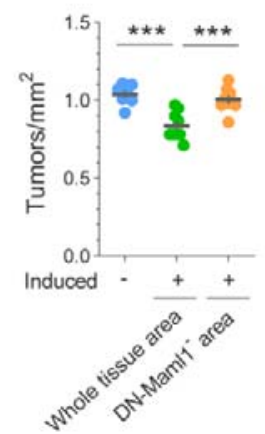

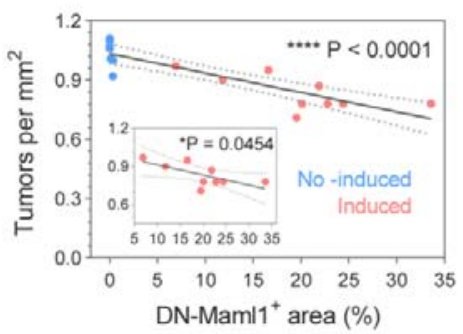

Extended Data Fig. 8. Expansion of highly competitive clones in normal tissue eliminates early tumors. (a) Cartoon illustrating the elimination of tumors due to competition with the highly competitive DN-Maml1 clones. Tumors are generated by DEN treatment followed by induction of DN-Maml1 clones with BNF and TAM. As DN-Maml1 clones expand they eliminate less fit tumors. (b) Qualitative representation of tumor dynamics following DN-Maml1 induction as compared to noninduced controls (see Supplementary information). (c) In vivo genetic lineage tracing using $A h c r e^{E R T} R o s a 26^{\text {Wt/DNM-GFP }}$ reporter mice. Upon injection of the drugs tamoxifen (TAM) and Bnapthoflavone (BNF), Cre-mediated recombination results in the heritable expression of the highly competitive dominant negative allele of Maml-1 fused to GFP fluorescent protein (DN-Mam/1), which will then be expressed in all the progeny of the single marked cells, generating clusters of labelled mutant clones. (d) Confocal image of a DEN-treated Ahcre ${ }^{E R T} R o s a 26^{\text {Wt/DNM-GFP }}$ mouse whole $\mathrm{EE}$, depicting DN-Maml1 clones (green). Mice were induced ten days after DEN withdrawal and esophagus collected twenty days later (as in Fig. 4b). Scale-bar $=2 \mathrm{~mm}$. (e) Percentage of EE area covered by DN-Maml1 clones in induced and non-induced (control) mice ( $n=9$ and 7 mice, respectively). Error-bars are Mean \pm s.e.m. ${ }^{* * *} \mathrm{P}<0.001$ (Two-sided Mann-Whitney test). (f) Number of tumors per $\mathrm{mm}^{2}$ of EE in control non-induced $(n=7)$ and induced $(n=9) A h c r e^{E R T} R O S a 26^{\text {wt/DNM-GFP }}$ mice. Data for the induced mice is show as the number of tumors per $\mathrm{mm}^{2}$ of whole EE (whole tissue area) and per $\mathrm{mm}^{2}$ of EE not covered by DN-Maml1 clones (DN-Maml1 area). Error-bars are Mean \pm s.e.m. ${ }^{* * *}<<0.001$ (Two-sided Mann-Whitney test). (g) Correlation between the area of EE covered by DN-Mam 1 clones and the number of tumors in induced and non-induced $A h c r e^{E R T} R O S a 26^{\text {Wt/DNM-GFP }}$ mice. Line shows the Pearson correlation $\left(R^{2}=0.7526\right)$ with $95 \%$ confidence interval (dotted lines). Inset shows the correlation of the induced mice only $\left(R^{2}=0.4577\right)$. 


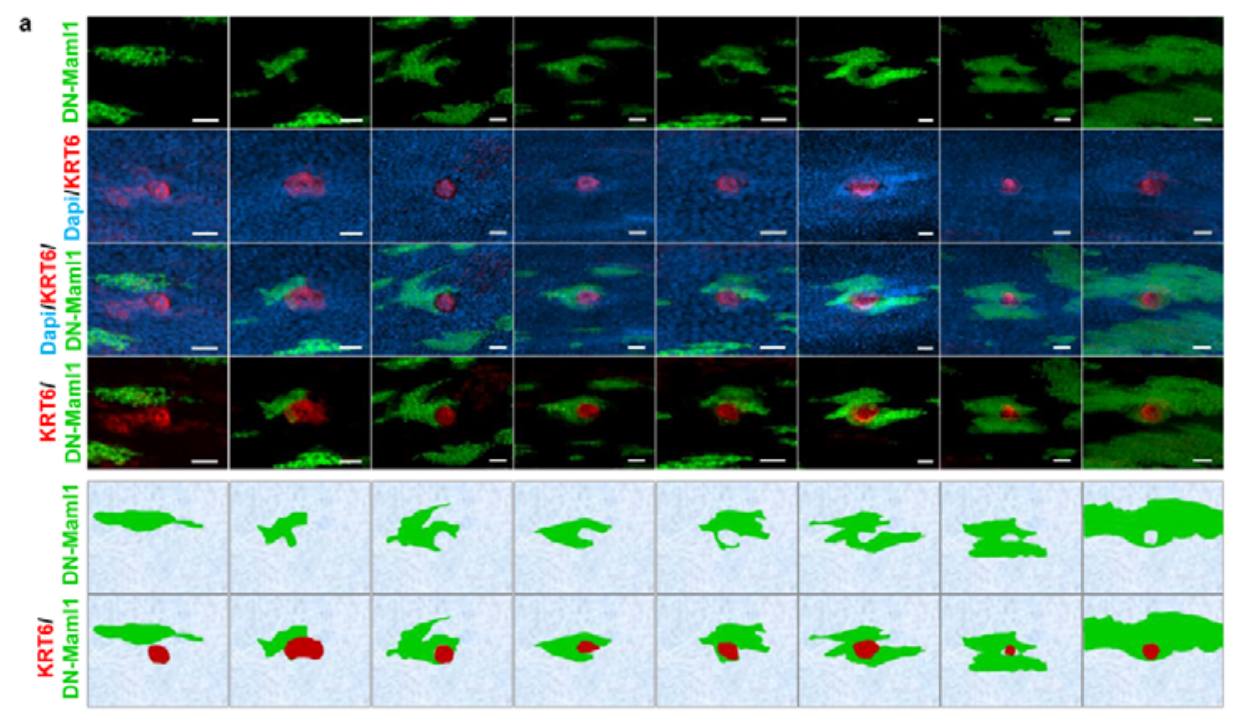

b
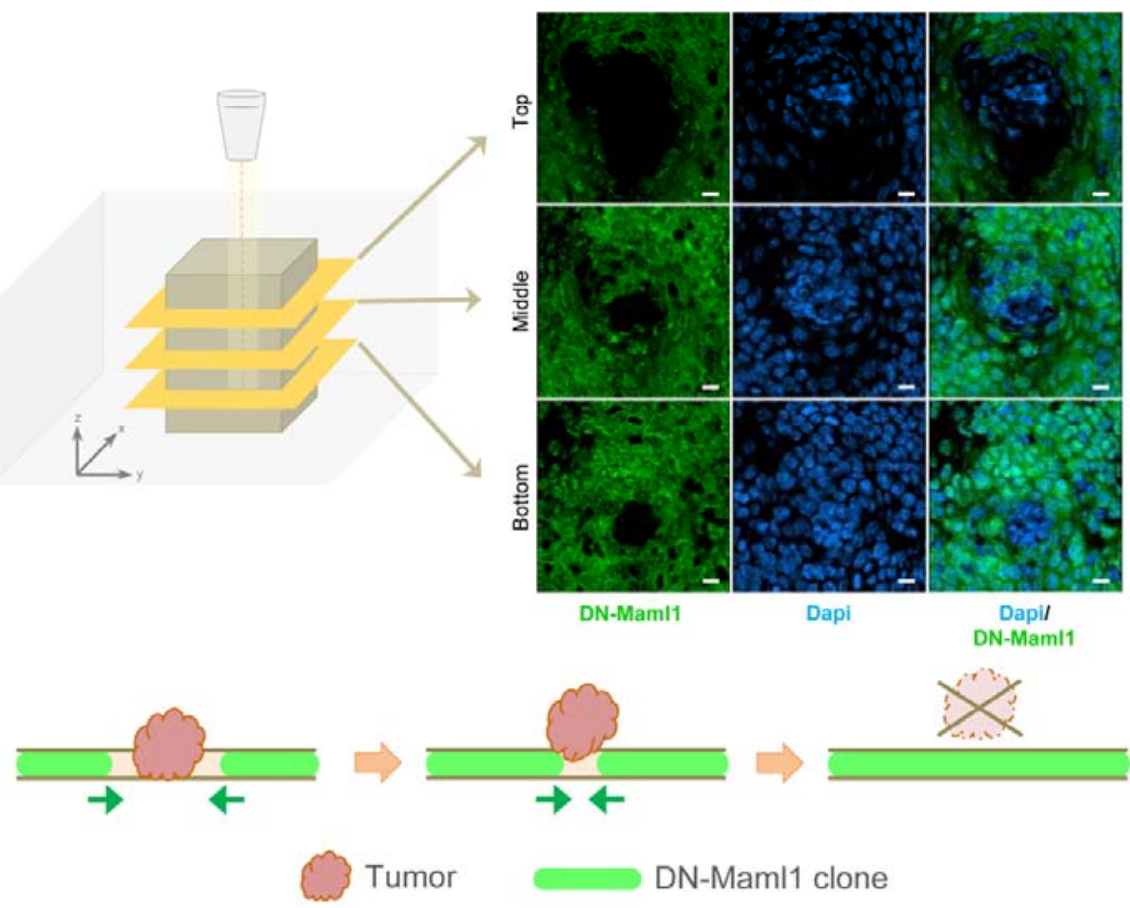

Extended Data Fig. 9. Spatial interaction between DN-Maml1 clones and tumors. (a) Confocal images and cartoons of EE from $A h c r e^{E R T} R O S a 26^{\text {wt/DNM-GFP }}$ mice treated with DEN for 2 months, induced 10-days post-DEN withdrawal and tissues collected 20-days later. The images show different steps of DN-Maml1 clones (green) interacting with early tumors (red), from barely touching (left) to completely surrounding the tumors (right). Scale-bars: $25 \mu \mathrm{m}$. (b) Top-down confocal images depicting a DN-Maml1 clone surrounding a tumor (see also Fig. 4e). Scale-bars: 10 $\mu \mathrm{m}$. A smaller tumor surface area at the base strongly suggest a process of extrusion by the expanding DN-Maml1 clone as illustrated in (c). 
bioRxiv preprint doi: https://doi.org/10.1101/2021.06.25.449880; this version posted June 25, 2021. The copyright holder for this preprint (which was not certified by peer review) is the author/funder, who has granted bioRxiv a license to display the preprint in perpetuity. It is made available under aCC-BY 4.0 International license.

a

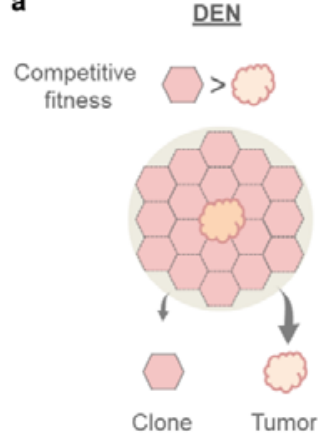

$\underline{D E N}+\mathrm{DBZ}$

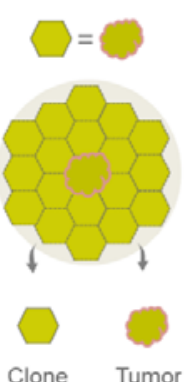

b

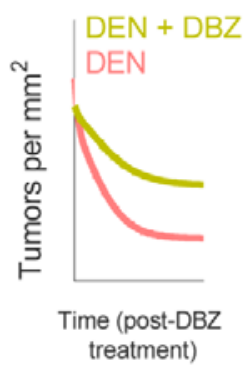

c

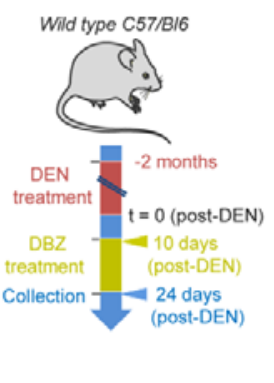

d

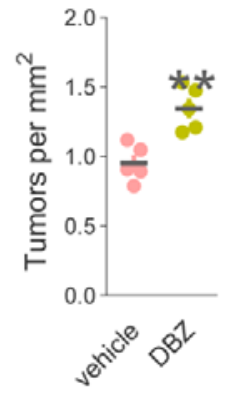

e

Human esophagus

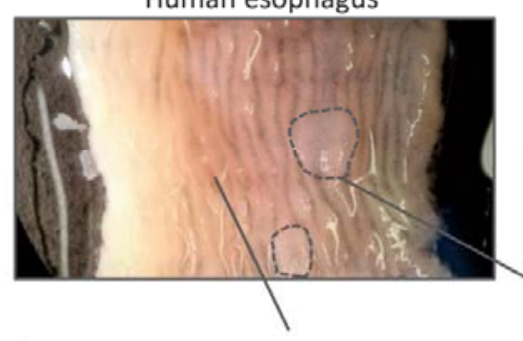

f

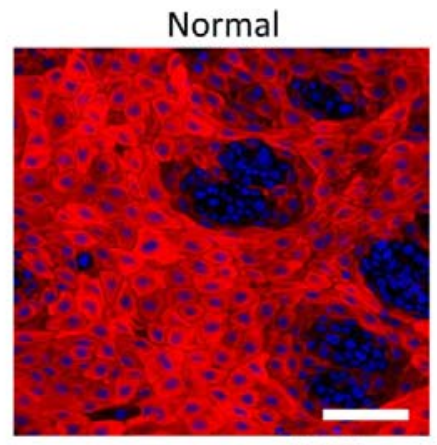

KRT6/Topro3
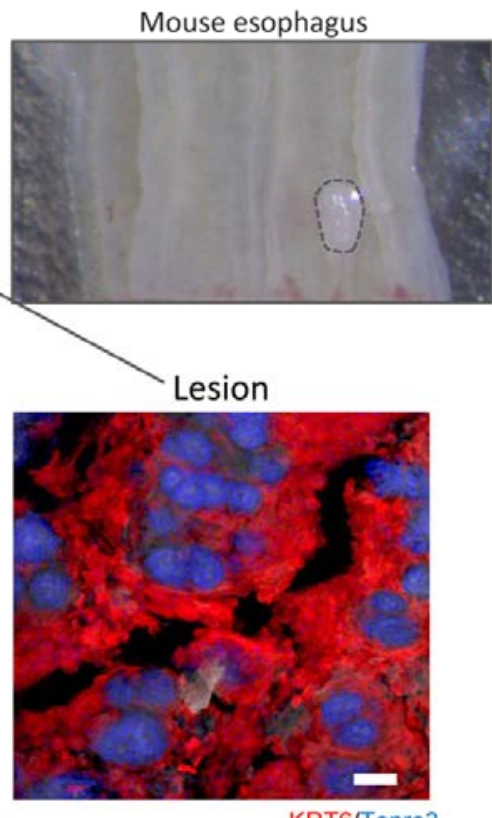

KRT6/Topro3

Extended Data Fig. 10. Increasing competitive fitness throughout EE slows down tumor loss. Human esophageal lesions. (a) Cartoon illustrating the predicted effects on tumor burden after leveling up the competitive fitness in normal and neoplastic EE through administration of DBZ. (b) Qualitative representation of tumor dynamics following DBZ treatment as compared to vehicle-treated controls. (c) Protocol: wild-type mice were treated with DEN for two months. Ten days after DEN withdrawal mice received DBZ or vehicle control and tissues were harvested two weeks later. (d) Number of tumors per $\mathrm{mm}^{2}$ of EE in DBZ and vehicle control treated mice. Error-bars are Mean \pm s.e.m. $* * \mathrm{P}=0.0079$ (Two-sided Mann-Whitney test, $\mathrm{n}=5$ mice per group). (e) Images of human (left) and mouse (right) esophagus. Tissues were longitudinally cut opened and flattened. Dotted lines delineate lesions. Scale-bars: $1 \mathrm{~mm}$. (f) Confocal images of normal (left) and neoplastic (right) epithelium stained with KRT6 (red) and Topro3 (nuclei, blue). Scale-bars: $100 \mu \mathrm{m}$. 